\title{
Channeling the final say in politics
}

\section{Working Paper}

\section{Author(s):}

Gersbach, Hans; Imhof, Stephan; Tejada, Oriol

Publication date:

2012-08

\section{Permanent link:}

https://doi.org/10.3929/ethz-a-007349802

\section{Rights / license:}

In Copyright - Non-Commercial Use Permitted

Originally published in:

Economics Working Paper Series 12/164(164) 


\title{
Channeling the Final Say in Politics*
}

\author{
Hans Gersbach \\ CER-ETH Center of Economic Research \\ at ETH Zurich and CEPR \\ 8032 Zurich, Switzerland \\ hgersbach@ethz.ch
}

\author{
Stephan Imhof \\ CER-ETH Center of Economic Research \\ at ETH Zurich \\ 8032 Zurich, Switzerland \\ stimhof@ethz.ch
}

\author{
Oriol Tejada \\ CER-ETH Center of Economic Research \\ at ETH Zurich \\ 8032 Zurich, Switzerland \\ toriol@ethz.ch
}

This Version: August 2012

\begin{abstract}
We examine how the final say in a sequence of proposals for local public project provision, financing, and redistribution can be channeled towards socially desirable outcomes, thereby breaking the dictatorial power of the last agendasetter. Individuals are heterogeneous with some citizens benefiting from the public project (winners) and the rest losing (losers) relative to per-capita costs. Our main insight is that a simple ban on subsidies for the proposal-makers can achieve the purpose whenever the first proposal-maker is a winner and the second proposal-maker is a loser. Such a ban induces project winners to make efficient public project proposals that are however coupled with socially undesirable subsidy schemes. The best possible amendment for project losers is then to match the project proposal and to eliminate all subsidies. We further show that two-round proposal-making constitutes the minimal form of political competition yielding first-best outcomes and that restrictions on tax schemes are socially desirable.
\end{abstract}

Keywords: constitutional design, local public project provision, subsidies, majority rule

JEL Classification: D72, H40

*We would like to thank Volker Hahn for valuable comments and suggestions. 


\section{Introduction}

Rules and procedures may have a strong impact on collective decisions. Since the classic results provided by McKelvey $(1976,1979)$, this fact has been emphasized for policy spaces that include flexible redistributional politics. For such policy spaces, a Condorcet winner does not typically exist. According to McKelvey, in such circumstances an agenda-setter can act as a dictator if individuals vote myopically in a sequence of proposals.

Berheim, Rangel, and Rayo (2006) show that the agenda-setter may not need to control the entire agenda to achieve her preferred outcome. In the case of pure redistribution proposals, for instance, the last proposer can always effectively dictate the outcome.

In this paper, we examine how the final say in a sequence of proposals can be channeled towards socially desirable outcomes. More specifically, we study local public project provision, financing, and flexible redistribution in a large electorate with risk-neutral citizens. Individual utilities of the public project are heterogeneous with some citizens benefiting from the public project - henceforth called winners - and the rest losing - henceforth called losers - relative to per-capita costs. Since we are concerned with local public projects, the share of winners is smaller than the share of losers. We further assume that there are small but positive deadweight costs of redistribution. Accordingly, a socially optimal allocation prescribes public-project provision if and only if aggregate benefits exceed production costs and there are no subsidies, i.e., taxes are solely raised for the financing of the public project.

We consider an agenda-setting sequence in which, first, a winner makes a proposal and, second, a loser makes an amendment. A proposal sets out whether or not to provide the public project, the tax rate, and an arbitrary distribution of (nonnegative) subsidies among the electorate. Once the two proposals have been made, all individuals cast a vote to choose one of the proposals. The decision is taken according to the simple majority rule.

Our major insight is that forbidding proposal-makers to channel subsidies to themselves yields first-best outcomes and breaks the dictatorial power of the last proposal-maker. Such a ban induces project winners to make efficient public project proposals coupled with subsidies to a fraction of the society. Although project losers may dislike the 
project proposal, their best possible amendment - attracting the support of a majority of citizens - is to match the project proposal and to eliminate all subsidies so that the final say is channeled toward a socially desirable outcome. A major part of the paper will be devoted to the proof of this result and to its significance.

We also show that, without further rules, the power of the last word in proposalmaking as identified in the literature fully applies. In particular, as a loser has the last word, she will desist from proposing the public project. Instead, she will propose an allocation of subsidies supported by a majority and benefiting herself most. However, when proposal-makers - in the first and the second round - are not allowed to offer subsidies to themselves (disallowance of agenda-setter subsidies), all inefficiencies in the collective process are avoided. Thus the say of the loser making the final proposal is channeled toward the socially desirable outcomes. In particular, at the final stage, we obtain that

- socially efficient public projects are proposed and accepted,

- socially inefficient public projects are not proposed,

- no subsidies are proposed.

A short version of the intuition for this result has been given above; an extended version will be set out in Section 5. In order to assess the robustness and significance of the results, we explore several variations of the political process and obtain the following additional results.

First, if no amendment is possible and only one winner makes a proposal, some inefficiencies may occur, even when subsidies for the agenda setter are not allowed. Thus, two-round proposal-making constitutes the minimal form of political competition that yields first-best results.

Second, allowing losers to move first and winners to make amendments does not produce first-best outcomes. Specifically, under the reversed-order two-round institution, the second agenda-setter, a winner, can often impose her preferred policy, regardless of whether the project is socially desirable.

The paper is organized as follows. In Section 2 we situate the paper in the context of the literature on the subject. Section 3 is devoted to presenting the formal model and 
the main assumptions. In Section 4 we present the two-round institution and show that, in the absence of rules that restrict subsidies to agenda-setters, the last agendasetter can effectively act as a dictator. Section 5 contains the main finding of the paper, which is that when subsidies to agenda-setters are banned, the final say yields the social optimum. In Section 6 we investigate the robustness of our main result by modifying some of the features of the two-round institution analyzed in the previous section. Section 7 concludes.

\section{Relation to the Literature}

Our paper is related to several strands of the literature. First, our model shares features of Bernheim, Rangel, and Rayo (2006) to the effect that an approved proposal in the first round becomes the default point for the second round, while real-time agenda setting occurs in which a second agenda-setter can condition her proposal on the first proposal and its voting outcome. There is a large literature on sequential agendas that include fixed and evolving default points, e.g. Shepste and Weingart (1984), Ferejohn, Fiorina, and McKelvey (1987), Romer and Rosenthal (1978), Austen-Smith (1987), Banks (1985), Dutta, Jackson, and Le Breton (2001), Banks and Gasmi (1987), and Miller (1980). Another strand of literature has focused on real-time agenda setting and fixed defaults (Baron-Ferejohn 1989, Banks and Duggan 2000, Diermeier and Merlo 2000). We adopt a normative approach and examine which type of agenda-setter rules and which amendment rule can channel the final say in politics toward socially desirable outcomes.

Second, we study how public-project provision and redistribution interact. Similar interactions have been studied by Bernheim, Rangel, and Rayo (2006) and Jackson and Moselle (2002). The latter extend the Baron and Ferejohn (1989) model with a closed rule by adding an ideological component. As the time horizon is infinite, dictatorial effects of the last proposal-maker do not arise. However, their finding that ideological and distributional issues cannot be separated, as redistribution helps to reach a compromise in the ideological issue, displays parallels with our article, in which public-project provision and redistribution are entangled. In our context, though, social efficiency requires zero redistribution, and we search for rules that simultaneously 
achieve this objective plus social efficiency regarding public-project provision.

Third, we consider legislative policy-making with given tax rules embodied in different laws. We assume that the financing of public-project provision and redistribution occurs solely through a uniform tax rate. Proposal-makers can, however, channel subsidies flexibly to different constituencies. The combination of tax rules and flexible subsidization in actual legislative policy-making is discussed and justified in Gersbach, Hahn, and Imhof (2012). While we take these characteristics of legislative policymaking as given for most of our analysis, in Section 6 we provide a further justification why it is socially desirable that flexibility in taxation and subsidization differs. Specifically, we show that first-best solutions are not attainable if proposal-makers can be fully flexible with respect to taxation and subsidization.

\section{Model}

\section{$3.1 \quad$ Set-up}

We consider a society facing the standard problem of local public-project provision and financing. Citizens are indexed by either $i$ or $j$ and are uniformly arranged on the unit interval. The provision of a public project yields benefits $v_{j}$ for all $j \in[0,1]$ and involves per-capita costs $k \geq 0$. For simplicity of exposition, we assume $v_{j} \in\left\{V_{w}, V_{l}\right\}$ with $V_{w}>$ $k>V_{l}{ }^{1}$ Accordingly, we refer to individuals obtaining $V_{w}$ from the public project as winners and to individuals receiving $V_{l}$ as losers. Without loss of generality, we assume winners to be located on the interval $[0, p]$ and losers on the interval $(p, 1]$. We assume throughout the paper that one feature of local public projects is that only a minority of the society benefits from them, that is, $p<1 / 2$. The set of all possible public projects comprises all quadruples $\left(V_{w}, V_{l}, k, p\right)$ that satisfy the assumptions introduced so far, i.e.

$$
\mathcal{P}:=\left\{\left(V_{w}, V_{l}, k, p\right) \in \mathbb{R}_{++} \times \mathbb{R} \times \mathbb{R}_{+} \times(0,1 / 2) \mid V_{w}>k>V_{l}\right\}
$$

For subsequent analysis, we consider an arbitrary public project from the set $\mathcal{P}$. The project parameters and the identities of winners and losers are common knowledge. In practice, individual valuations may be more or less known to the electorate as a whole.

\footnotetext{
${ }^{1}$ Note that $V_{l}$ may be higher or lower than zero.
} 
Typical examples of public projects in which winners and losers of a public project are common knowledge are building roads, closing down military bases, reducing subsidies to the agricultural sector, or improving a school building.

A proposal, denoted by $\pi$, comprises a subsidy scheme and a decision on the public project. Subsidies are constrained to be non-negative and bounded from above by $\hat{s} \in \mathbb{R}^{+}$. We assume that $\hat{s}>V_{w}$, so agents can be subsidized quite generally. ${ }^{2}$ Let $\mathbb{S}$ be the set of all non-negative and Lebesgue-measurable and Lebesgue-integrable functions on the unit interval. Each subsidy scheme involved in a proposal $\pi$ is a function $s(\pi) \in \mathbb{S}$. We use $s_{j}(\pi)$ to denote the subsidy given to individual $j \in[0,1]$. The variable $g(\pi) \in\{0,1\}$ indicates whether the public project is suggested $(g(\pi)=1)$ or not $(g(\pi)=0)$ under proposal $\pi$. Accruing costs are financed through a uniform tax determined by the society's budget constraint:

$$
g(\pi) k+(1+\varepsilon) \int_{0}^{1} s_{j}(\pi) d j=t(\pi)
$$

In (1), $t(\pi)$ represents the per-capita tax, which is uniquely determined by $g(\pi)$ and the subsidy scheme $s(\pi)$. In the main body of the paper we work with a rule that taxes all individuals uniformly. But in Section 6 we explore the significance of the presence of tax rules by allowing arbitrary taxation of individuals. We also assume that deadweight costs of redistribution, captured by $\varepsilon>0$ in (1), are small but positive. ${ }^{3}$ To sum up, the set of possible proposals is

$$
\Pi:=\left\{\pi=(g(\pi), s(\pi)) \in\{0,1\} \times \mathbb{S} \mid \begin{array}{r}
g(\pi) k+(1+\varepsilon) \int_{0}^{1} s_{j}(\pi) d j=t(\pi) \\
\text { and } s_{j}(\pi) \leq \hat{s} \text { for all } j \in[0,1]
\end{array}\right\} .
$$

In our setting, an institution is a set of rules the application of which yields a proposal that will be implemented. A relevant political problem is then how to design an institution that always induces implementation of a welfare-maximizing proposal.

In the present paper we consider an institution based on a sequential political competition. More precisely, the sequence of events is as follows:

\footnotetext{
${ }^{2}$ If subsidies were unbounded, a small subset of agents could fully exploit the entire electorate.

${ }^{3}$ Deadweight costs of redistribution are frequently used in the literature (see Aghion and Bolton 2003, Gersbach 2009, or Gersbach, Hahn and Imhof 2012). Such costs can be interpreted in a narrow sense, e.g. as resources used for collecting and transferring funds. In a broader sense, they may represent distortions, say when individuals reduce labor supply because income is taxed.
} 
(1) A winner suggests a proposal. Without loss of generality, we assume this winner is $j=0$, and correspondingly use $\pi^{0}$ to denote her proposal.

(2) A loser suggests a counter-proposal. Without loss of generality, we assume this loser is $j=1$, and correspondingly use $\pi^{1}$ denote her proposal.

(3) All individuals cast a vote on either $\pi^{0}$ or $\pi^{1}$, and no abstention occurs. ${ }^{4}$ The proposal that receives more votes is adopted. ${ }^{5}$ Ties between proposals are broken in favor of the counter-proposal (see Tie-Breaking Rule 2 below).

For notational clarity we will refer to $j=0$ as $A S_{w}$ (agenda-setter who is a winner) and to $j=1$ as $A S_{l}$ (agenda-setter who is a loser).

Adoption of a proposal $\pi \in\left\{\pi^{0}, \pi^{1}\right\}$ yields to a citizen $j \in[0,1]$ a net utility change $u_{j}(\pi)$ given by

$$
u_{j}(\pi)=g(\pi) v_{j}+s_{j}(\pi)-t(\pi) \text { for } j \in[0,1] .^{6}
$$

Accordingly, the utilitarian welfare measure associated with a proposal $\pi \in\left\{\pi^{0}, \pi^{1}\right\}$ amounts to

$$
W(\pi)=g(\pi)\left[p V_{w}+(1-p) V_{l}-k\right]-\varepsilon \int_{0}^{1} s_{j}(\pi) d j .
$$

For the remainder of the paper, it is sufficient to make $\varepsilon$ arbitrarily small but to keep in mind that paying out subsidies lowers aggregate welfare. This will greatly facilitate the presentation.

\subsection{Socially efficient solution}

We next characterize the socially efficient solution chosen by a social planner who maximizes the utilitarian welfare for any given realization of parameters $\left(V_{w}, V_{l}, k, p\right) \in$ $\mathcal{P}$. Such a social planner will implement the public project if and only if it is socially efficient, i.e., if and only if $p V_{w}+(1-p) V_{l} \geq k$. Moreover, she will never choose

\footnotetext{
${ }^{4} \mathrm{~A}$ weaker assumption for which all the results in this paper hold is that abstention exogenously affects losers and winners in the same proportion. More generally, it would suffice to require the share of "active" losers to be larger than the share of "active" winners, "active" here referring to individuals who vote according to an exogenous decision.

${ }^{5}$ Such a setting corresponds to the legal system of direct democracy or parliamentary democracy with perfect representation.

${ }^{6}$ We assume that individuals' income is sufficient to pay taxes under any proposal considered.
} 
a positive level of total subsidies as it would reduce welfare. These observations are summarized in the following definition.

\section{Definition 1}

A socially optimal proposal $\pi^{s o c} \in \Pi$ has the following characteristics:

$$
g\left(\pi^{s o c}\right)=\left\{\begin{array}{ll}
1 & \text { if } p V_{w}+(1-p) V_{l} \geq k, \\
0 & \text { if } p V_{w}+(1-p) V_{l}<k,
\end{array} \quad \text { and } \quad s_{j}\left(\pi^{s o c}\right)=0 \text { for all } j \in[0,1]\right.
$$

In this paper we adopt the incomplete social contract perspective (see Aghion and Bolton 2003, Gersbach 2009). In particular, although project parameters and valuations are observable, they are not verifiable in court. If parameters were verifiable, complete social contracts guaranteeing socially optimal solutions could be drafted. As a consequence, constitutions can only contain rules on how to decide on public projects. As we are considering the democratic provision of public projects (Gersbach 2009), we are looking at collective choice processes in which agenda setters make proposals on which citizens vote according to majority rule.

\subsection{Tie-breaking rules}

We impose the following tie-breaking rules to simplify the presentation.

\section{Tie-Breaking Rule 1}

If $u_{j}\left(\pi^{0}\right)=u_{j}\left(\pi^{1}\right)$, individual $j$ will vote for the counter-proposal $\pi^{1}$.

\section{Tie-Breaking Rule 2}

If one half of the society votes in favor of $\pi^{0}$ and the other half in favor of $\pi^{1}$, the counter-proposal $\pi^{1}$ will be implemented.

\section{Tie-Breaking Rule 3}

If $A S_{l}$ is indifferent between a proposal $\pi^{1} \in \Pi$ involving $g\left(\pi^{1}\right)=1$ and a proposal $\pi^{\prime 1} \in \Pi$ involving $g\left(\pi^{\prime 1}\right)=0$, she will always suggest $\pi^{1}$.

Tie-Breaking Rules 1 and 2 enable the second agenda-setter to ensure that the counterproposal is adopted if at least half of the electorate are no worse off than they would be with the first proposal. Together with Tie-Breaking Rule 3, these two rules simply facilitate presentation and do not affect our results. Without them, we would need to discretize the strategy spaces. 


\subsection{Types of inefficiency}

Given an arbitrary institution, there are three types of inefficiency that may arise in the process of choosing a proposal:

1. Socially inefficient public projects may be implemented.

2. Socially efficient public projects may not be implemented.

3. Socially harmful subsidies may arise.

As we show in this paper, avoiding all three types of inefficiency can be achieved by sequential political competition as long as it is supplemented by appropriate subsidy rules. Moreover, as soon as the design features of that institution are changed, inefficiencies will emerge (see Section 6).

\section{The (Undesirable) Power of the Last Say}

In this section we show that results on "the power of the last word" (Bernheim, Rangel and Rayo 2006, and Myerson 1993) are also valid in our context if no further rules are imposed. For this purpose, we define the indicator function $I\left(\pi^{0}, \pi^{1}\right)$, which adopts a value of 1 if proposal $\pi^{1}$ is implemented and 0 if proposal $\pi^{0}$ is implemented:

$$
I\left(\pi^{0}, \pi^{1}\right)= \begin{cases}1 & \text { if } u_{j}\left(\pi^{1}\right) \geq u_{j}\left(\pi^{0}\right) \text { holds for at least half of the society } \\ 0 & \text { otherwise }\end{cases}
$$

Observe that the above definition implicitly uses Tie-Breaking Rules 1 and 2. In our setting, the power of the last word means that the second agenda-setter, $A S_{l}$, can always obtain approval for the proposal that is best for her.

In order to analyze the dynamics of the proposed two-round institution, we apply the concept of subgame-perfect Nash equilibrium. Lemma 1 below substantially simplifies the analysis as counter-proposalsthat are not seconded by at least half of the society are ruled out in equilibrium.

\section{Lemma 1}

If a counter-proposal $R\left(\pi^{0}\right) \in \Pi$ is a best response to proposal $\pi^{0} \in \Pi$, then

$$
I\left(\pi^{0}, R\left(\pi^{0}\right)\right)=1
$$




\section{Proof:}

Given an arbitrary proposal $\pi^{0} \in \Pi$, the loser can always match the winner's proposal, i.e., choose $\pi_{C}^{1}:=\pi^{0}$. Since $u_{j}\left(\pi_{C}^{1}\right)=u_{j}\left(\pi^{0}\right)$ for all $j \in[0,1]$, it holds by Tie-Breaking Rule 1 that $I\left(\pi^{0}, \pi_{C}^{1}\right)=1$. Moreover, due to Tie-Breaking Rule 3, the loser will prefer making proposal $\pi_{C}^{1}$ to any proposal $\pi^{1} \neq \pi_{C}^{1}$ such that $I\left(\pi^{0}, \pi^{1}\right)=0$. As a consequence, either the loser will choose $\pi_{C}^{1}$ or she will choose a proposal $\pi^{1} \neq \pi_{C}^{1}$ such that $I\left(\pi^{0}, \pi^{1}\right)=1$ and $u_{1}\left(\pi^{1}\right) \geq u_{1}\left(\pi_{C}^{1}\right)=u_{1}\left(\pi^{0}\right) \cdot{ }^{7}$

As a consequence of Lemma 1 , the best-response function for $A S_{l}$ can be described by the correspondence $R: \Pi \rightarrow \Pi$ defined for any $\pi^{0} \in \Pi$ by

$$
R\left(\pi^{0}\right) \in \underset{\pi^{1} \in \Pi, I\left(\pi^{0}, \pi^{1}\right)=1}{\operatorname{argmax}}\left\{s_{1}\left(\pi^{1}\right)+g\left(\pi^{1}\right)\left(V_{l}-k\right)-\int_{0}^{1} s_{j}\left(\pi^{1}\right) d j\right\} .
$$

However, whenever the best response is uniquely defined up to a relabeling of individuals and redistribution within sets of Lebesgue-measure zero, we will slightly abuse our notation and consider $R(\cdot)$ to be a function rather than a correspondence.

We further assume that the second agenda-setter is well aware of the fact that her choice influences the choice of the first agenda-setter. In Proposition 1 below we show that, since redistribution within groups of Lebesgue-measure zero does not have an impact on the outcome in a continuum, the second agenda-setter, $A S_{l}$, can credibly condition subsidies to the first agenda-setter, $A S_{w}$, on a specific first proposal and in this way regulate the choice of $A S_{w}$.

\section{Proposition 1}

The outcome under the simple two-round procedure - proposal by a winner, counterproposal by a loser - may cause inefficiencies of type 2.

Proposition 1, the proof of which is to be found in Appendix A, illustrates that the second agenda-setter has an incentive to offer the first agenda-setter a subsidy $\hat{s}$ if the latter does not propose implementation of the public project and does propose zero

\footnotetext{
${ }^{7}$ There often exist more sophisticated counter-proposals $R\left(\pi^{0}\right)$ than a simple copy of $\pi^{0}$ that yield $u_{1}\left(R\left(\pi^{0}\right)\right)>u_{1}\left(\pi^{0}\right)$ and $I\left(\pi^{0}, R\left(\pi^{0}\right)\right)=1$. Investigation of such counter-proposals is part of our subsequent analysis.
} 
taxes. As a consequence, preserving the status quo and subsidization solely of agendasetters is a subgame perfect equilibrium. More particulary, in this equilibrium efficient projects are not implemented.

\section{Agenda-Setter Rules and the Last Say}

The negative result in Proposition 1 is predicated on the absence of rules for subsidization. We now introduce a very simple subsidization rule, which we call Agenda-Setter Rule, that will turn out to be sufficient to achieve socially efficient solutions.

\section{Agenda-Setter Rule}

A feasible proposal $\pi \in\left\{\pi^{0}, \pi^{1}\right\}$ has to comprise $s_{0}(\pi)=s_{1}(\pi)=0$.

This rule bans subsidies for agenda-setters completely. The main result of this paper - Theorem 1 below - shows that together with the Agenda-Setter Rule political competition ensures that the social optimum is always implemented.

\section{Theorem 1}

The equilibrium outcome under the simple two-round procedure-proposal by a winner, counter-proposal by a loser and voting with the Agenda-Setter Rule - is $\pi^{\text {soc } 8}{ }^{8}$

Accordingly, the equilibrium outcome equals the socially efficient outcome, so the simple two-round institution proposed in this paper inhibits all three types of inefficiency. The proof of the theorem is to be found in Appendix B. Here we provide an intuitive explanation in several stages. First, we introduce a special proposal: the public project is proposed and all members of the coalition supporting it obtain the same per capita utility. This utility is equal to the utility of a winner if the project were provided without subsidies. With such a proposal this means that subsidized losers will receive comparatively high subsidies.

Second, suppose momentarily that implementing the public project is socially efficient and the proposal outlined above is made by the first proposal-maker. Since all citizens have to pay the same taxes, a loser as second proposal-maker faces a difficult choice. In

\footnotetext{
${ }^{8}$ We note that proposals may differ with regard to a set of agents with measure zero.
} 
order to avoid the implementation of the public project and to lower the tax burden, the loser has to offer high subsidies to a fraction of losers subsidized under the first proposal in order to gain their support for the amendment. Alternatively, the second proposalmaker can reduce her tax burden by matching the first public project proposal and by eliminating all subsidies. Such a proposal will attract a majority of voters composed of all winners and all losers not receiving subsidies under the first proposal.

Third, if the public project is socially efficient, the latter route turns out to be more attractive for project losers and anticipating the response by the second proposal-maker the first proposal-maker will make the proposal outlined as point one.

Fourth, if the public project is socially inefficient, i.e., per capita gains are lower than per capita losses, the second proposal-maker will never find it profitable to propose project implementation. The minimal amount of subsidies necessary to form a coalition of winners and losers that supports the status quo will lead to the lowest possible tax burden. Since the first proposal-maker anticipates this behavior, she will prefer to abandon the public project and eliminate all subsidies in her proposal.

\section{Ramifications}

We have shown that socially desirable outcomes can always be achieved through the two-round institution analyzed so far in this paper. Recall that the defining features of the institution are:

- Subsidies to agenda-setters are restricted by rules.

- There are two rounds of proposals.

- Subsidies to non-agenda-setter voters are only restricted to be non-negative. ${ }^{9}$

- Taxes are imposed uniformly across voters.

- A winner proposes first, and a loser makes an amendment.

The present section is devoted to analyzing the robustness of our main result (Theorem 1) by dropping some of the above features. We have already seen (Proposition 1) that

\footnotetext{
${ }^{9}$ We have also assumed that subsidies are bounded from above. Otherwise, an agenda-setter could exploit the whole electorate.
} 
in the absence of agenda-setter rules and keeping all other features unchanged, the proposed institution will fail to invariably yield the socially efficient outcome. We now discuss what happens if other characteristics of the institution are changed.

First, we look at one-round institutions. We will show that under such circumstances, social inefficiencies may arise even if the Agenda-Setter Rule is imposed. ${ }^{10}$ This result implies that the two-round institution analyzed in Theorem 1 is minimal, where minimality refers to the number of rounds needed to ensure that the socially optimal outcome is always achieved. Minimality of the institution is a desirable property as long as there are (monetary or opportunity) costs associated with the preparation of proposals and voting procedures.

Second, we show that reversing the order of proposers (a loser proposes first and then a winner counter-proposes) may yield socially inefficient outcomes as well. As a consequence, we can say that the institution analyzed in Theorem 1 is unique among the set of minimal institutions that always yield the social optimum.

Third, we demonstrate that allowing for flexible tax systems instead of uniform taxation introduces inefficiencies.

In conclusion, the exact design of the two-round institution introduced in the paper is very robust, meaning that changing any of its defining features may lead to socially inefficient outcomes. The proofs of all the results presented in this section are given in Appendix C.

\subsection{Minimal form of political competition}

Gersbach, Hahn, and Imhof (2012) consider an institution that does not permit political competition. More precisely, they assume that only a winner can make a proposal. Accordingly, loser have no power of amendment. Throughout this subsection we consider the same set-up: a one-shot procedure with uniform taxation and arbitrary subsidy schemes plus the Agenda-Setter Rule. We obtain the following result.

\section{Proposition 2}

Under the one-shot procedure - proposal by a winner and voting with the Agenda-Setter

\footnotetext{
${ }^{10}$ Moreover, as already shown by Gersbach, Hahn, and Imhof (2012), this negative result cannot be rectified by further restricting the set of possible subsidy schemes.
} 
Rule - inefficiencies of types 1 and 3 may arise. ${ }^{11}$

In the next step we prove that limiting flexibility in the subsidy scheme does not prevent social inefficiencies. Let $\mathcal{A} \subseteq \mathbb{S}$ be any non-empty set of Lebesgue-measurable and Lebesgue-integrable functions on the unit interval bounded from above by $\hat{s}$. We assume that subsidy schemes must be chosen from $\mathcal{A}$.

\section{Proposition 3}

Under the modified one-shot procedure - proposal by a winner, voting with the AgendaSetter Rule, and limited subsidy schemes - inefficiencies of types 1 and 3 or inefficiencies of type 2 may arise.

It is straightforward to see that when the agenda-setter is a loser in a one-shot procedure, she will always choose not to implement the project under the Agenda-Setter Rule.

\subsection{Reverse order of proposal-making}

One might be tempted to think that any kind of political competition will preclude all types of inefficiency. There are four different possibilities of implementing a two-shot procedure depending on who proposes first (either a loser or a winner) and who makes a counter-proposal (either a loser or a winner). For obvious reasons, the only two significant sequences are winner/loser and loser/winner. ${ }^{12}$ Whereas the former option is efficient under the Agenda-Setter Rule, the second option fails to accomplish the same objectives. Accordingly, it is socially unprofitable to give political initiative to project losers, even when this option is feasible.

\section{Proposition 4}

Under the reversed two-shot procedure - proposal by a loser, counter-proposal by a winner, and voting with the Agenda-Setter Rule - inefficiencies of types 1 and 3 may arise.

\footnotetext{
${ }^{11}$ We note that inefficiencies of type 2 do not occur.

${ }^{12}$ The sequences winner/winner and loser/loser may yield socially inefficient outcomes. Moreover, they disallow one group from making a proposal and thus violate the principle of equal rights for proposal-making.
} 
The intuition for this result is as follows. For some combinations of $V_{w}$ and $V_{l}$ and when per capita costs of implementing a project are not very large, it is a best response for the second agenda-setter, $A S_{w}$, to subsidize some losers so as to gain their vote in favor of the implementation of the project. By doing so, she leaves the first agendasetter, $A S_{l}$, who anticipates $A S_{w}$ 's behavior, with no option but to propose project implementation in exchange for minimizing the amount of subsidies, which have to be paid according to a uniform tax.

\subsection{Flexible taxation}

For the main result of the paper we have assumed that subsidies can be distributed arbitrarily, whereas taxes are uniform. ${ }^{13}$ This reflects the widespread observation that there is much more flexibility in subsidy schemes than in tax systems (see Gersbach, Hahn and Imhof 2012). Nevertheless, from a conceptual point of view, restricting tax rules has to be justified. In Proposition 5 below we investigate the robustness of our results regarding the possibility of choosing arbitrary tax schemes.

\section{Proposition 5}

Under the flexible-taxation two-shot procedure-proposal by a winner, counter-proposal by a loser, flexible taxation, and voting with the Agenda-Setter Rule - inefficiencies of types 1 and 3 may arise.

Gersbach, Hahn, and Imhof (2012) show that restrictions on tax systems are advantageous for the society in one-shot procedures. Proposition 5 extends this insight to competitive political environments. This provides a further important justification for the use of tax rules in public-project provision.

\section{Conclusion}

In this paper we have advanced a simple rule that channels the final say in politics toward socially desirable outcomes. Numerous extensions deserve further scrutiny.

\footnotetext{
${ }^{13}$ Restrictions on tax distribution can be imposed in many other ways than uniform taxation, e.g. making the tax burden of agenda-setters dependent on the aggregate tax burden. Nevertheless, investigation of such rules is beyond the scope of the present paper and represents a subject for further research.
} 
Multiple or divisible public projects and income heterogeneity are two particularly important avenues for future research to explore. The present result may be a useful starting point for such endeavors. 


\section{Appendix A}

This section is entirely devoted to the proof of Proposition 1.

Proof of Proposition 1: Consider an arbitrary proposal $\pi^{0} \in \Pi$. Suppose that $\pi_{*}^{1} \in \Pi$ is a best response to $\pi^{0}$. We claim that proposals

$$
\begin{aligned}
& \tilde{\pi}^{1}:=\left(g\left(\pi_{*}^{1}\right), s\left(\tilde{\pi}^{1}\right)\right), \text { where } s_{j}\left(\tilde{\pi}^{1}\right)=s_{j}\left(\pi_{*}^{1}\right) \text { for all } j \in(0,1] \text { and } s_{0}\left(\tilde{\pi}^{1}\right)=\hat{s}, \\
& \tilde{\tilde{\pi}}^{1}:=\left(g\left(\pi_{*}^{1}\right), s\left(\tilde{\tilde{\pi}}^{1}\right)\right), \text { where } s_{j}\left(\tilde{\tilde{\pi}}^{1}\right)=s_{j}\left(\pi_{*}^{1}\right) \text { for all } j \in(0,1] \text { and } s_{0}\left(\tilde{\tilde{\pi}}^{1}\right)=0
\end{aligned}
$$

are also best responses to $\pi^{0}$. To see this, note that $\tilde{\pi}^{1}, \tilde{\tilde{\pi}}^{1} \in \Pi$ and, compared to $\pi_{*}^{1}$, only redistribution within measure zero groups is carried out, which implies $t\left(\tilde{\pi}^{1}\right)=$ $t\left(\tilde{\tilde{\pi}}^{1}\right)=t\left(\pi_{*}^{1}\right)$ and $u_{j}\left(\tilde{\pi}^{1}\right)=u_{j}\left(\tilde{\tilde{\pi}}^{1}\right)=u_{j}\left(\pi_{*}^{1}\right)$ for all $j \in(0,1]$. Consequently, $I\left(\pi^{0}, \tilde{\pi}^{1}\right)=$ $I\left(\pi^{0}, \tilde{\tilde{\pi}}^{1}\right)=I\left(\pi^{0}, \pi_{*}^{1}\right)=1$, where the last equality holds by Lemma 1 . Therefore all three proposals yield the same utility for $A S_{l}$, which confirms the claim.

Now suppose that $A S_{l}$ chooses $\pi^{1}$ according to the following rule: ${ }^{14}$

$$
\pi^{1}= \begin{cases}\tilde{\pi}^{1} & \text { if } \pi^{0} \text { is such that } g\left(\pi_{*}^{1}\right)=0, s_{1}\left(\pi_{*}^{1}\right)=\hat{s}, \text { and } t\left(\pi_{*}^{1}\right)=0, \\ \tilde{\pi}^{1} & \text { otherwise. }\end{cases}
$$

For $A S_{w}$, this implies that, for any choice of $\pi^{0}$ :

$$
u_{0}\left(\pi^{1}\right)= \begin{cases}u_{0}\left(\tilde{\pi}^{1}\right)=\hat{s} & \text { if } g\left(\pi_{*}^{1}\right)=0, s_{1}\left(\pi_{*}^{1}\right)=\hat{s}, t\left(\pi_{*}^{1}\right)=0, \\ u_{0}\left(\tilde{\tilde{\pi}}^{1}\right)=g\left(\tilde{\pi}^{1}\right) V_{w}-t\left(\tilde{\pi}^{1}\right) & \text { otherwise. }\end{cases}
$$

As $\hat{s}>V_{w}$, we obtain

$$
u_{0}\left(\tilde{\pi}^{1}\right)>V_{w} \geq u_{0}\left(\tilde{\tilde{\pi}}^{1}\right) .
$$

Thus, in equilibrium, the public project will not implemented, since $A S_{w}$ will prefer to abandon the public project and receive high subsidies in return by proposing a suitable chosen $\pi^{0}$ such that $\pi_{*}^{1}$, the best response to $\pi^{0}$, satisfies $g\left(\pi_{*}^{1}\right)=0, s_{1}\left(\pi_{*}^{1}\right)=\hat{s}$, $t\left(\pi_{*}^{1}\right)=0$.

Finally observe that the rule in (3) is optimal for $A S_{l}$ because it guarantees her the highest possible utility, that is

$$
u_{1}\left(\tilde{\pi}^{1}\right)=\hat{s} \geq u_{1}\left(\pi^{1}\right) \text { for all } \pi^{1} \in \Pi .
$$

\footnotetext{
${ }^{14}$ Note that, when only a subset of agents with zero measure is subsidized, the average tax burden of the entire society vanishes.
} 


\section{Appendix B}

In this section we prove Theorem 1. For this purpose, we first examine individual voting behavior and the second agenda-setter's best response. Then, we find the optimal strategy for the first agenda-setter.

\section{B.1 Individual voting behavior}

Let $\pi^{0}$ and $\pi^{1}$ be two arbitrary proposals in $\Pi$. Under Tie-Breaking Rule 1, individual $i \in[0,1]$ will vote in favor of proposal $\pi^{1}$ if and only if $u_{i}\left(\pi^{1}\right) \geq u_{i}\left(\pi^{0}\right)$, which can be rewritten as

$$
s_{i}\left(\pi^{1}\right) \geq s_{i}\left(\pi^{0}\right)+\left(g\left(\pi^{0}\right)-g\left(\pi^{1}\right)\right)\left(v_{i}-k\right)-\left(\int_{0}^{1} s_{j}\left(\pi^{0}\right) d j-\int_{0}^{1} s_{j}\left(\pi^{1}\right) d j\right) .
$$

Given a proposal $\pi^{0}=\left(g\left(\pi^{0}\right),\left(s_{j}\left(\pi^{0}\right)\right)_{j \in[0,1]}\right)$ and subsidies of the counter-proposal $\left(s_{j}\left(\pi^{1}\right)\right)_{j \in[0,1] \backslash\{i\}}$ to all individuals except $i$, it will prove very useful to define

$$
\sigma_{i}\left(g\left(\pi^{1}\right)\right):=s_{i}\left(\pi^{0}\right)+\left(g\left(\pi^{0}\right)-g\left(\pi^{1}\right)\right)\left(v_{i}-k\right)-\left(\int_{0}^{1} s_{j}\left(\pi^{0}\right) d j-\int_{0}^{1} s_{j}\left(\pi^{1}\right) d j\right) .
$$

Note that $\sigma_{i}\left(g\left(\pi^{1}\right)\right)$ represents the smallest subsidy that must be paid to individual $i$ so that she will vote for proposal $\pi^{1}$ given $g\left(\pi^{1}\right)$ and subsidies for all individuals $j \in[0,1]$, $j \neq i$, under $\pi^{1}$. In particular, if $\sigma_{i}\left(g\left(\pi^{1}\right)\right) \leq 0$, individual $i$ will vote for proposal $\pi^{1}$ irrespective of the size of the subsidy she receives under $\pi^{1}$.

Whereas $\sigma_{i}\left(g\left(\pi^{1}\right)\right)$ for each $i \in[0,1]$ may vary depending on the entire proposals $\pi^{0}$ and $\pi^{1}$, we notice that, for any $i, j \in[0,1]$ with $i \neq j, \sigma_{i}\left(g\left(\pi^{1}\right)\right)-\sigma_{j}\left(g\left(\pi^{1}\right)\right)$ depends solely on $s_{i}\left(\pi^{0}\right), s_{j}\left(\pi^{0}\right), g\left(\pi^{0}\right)$, and $g\left(\pi^{1}\right)$. Let $i, j \in[0,1]$ be two arbitrary different individuals. Then,

$$
\sigma_{j}\left(g\left(\pi^{1}\right)\right)-\sigma_{i}\left(g\left(\pi^{1}\right)\right)=s_{j}\left(\pi^{0}\right)-s_{i}\left(\pi^{0}\right)+\left(g\left(\pi^{0}\right)-g\left(\pi^{1}\right)\right)\left(v_{j}-v_{i}\right)
$$

In particular, given $\pi^{0}=\left(g\left(\pi^{0}\right), s\left(\pi^{0}\right)\right) \in \Pi$ and $g\left(\pi^{1}\right) \in\{0,1\}$, the sign of $\sigma_{j}\left(g\left(\pi^{1}\right)\right)-$ $\sigma_{i}\left(g\left(\pi^{1}\right)\right)$ for any two individuals $i, j \in[0,1], i \neq j$, is not affected by the choice of the subsidy scheme $s\left(\pi^{1}\right)$. 


\section{B.2 The best response of the second agenda-setter}

According to (2), the best response function for $A S_{l}$ is described by a correspondence $R(\cdot)$ from $\Pi$ into itself. However, when the best response is uniquely defined up to a relabeling of individuals and redistribution within sets of Lebesgue-measure zero, it will turn out to be more convenient to consider $R(\cdot)$ to be a function rather than a correspondence.

For the analysis of the second mover's strategy, it is useful to introduce the following sets: ${ }^{15}$

- $N:=\left\{j \in(p, 1) \mid s_{j}\left(\pi^{0}\right)=0\right\}$

- $\bar{N}:=\{j \in(0,1) \mid j \notin N\}$.

Observe that $N$ is the set of all losers, except $A S_{l}$, who receive zero subsidies under the proposal $\pi^{0}$, whereas $\bar{N}$ is the set of all individuals, except $A S_{w}$ and $A S_{l}$, who do not belong to $N$. For an arbitrary set $M \subseteq[0,1]$, let the corresponding lower-case letter, $m$, denote its Lebesgue measure. It is obvious that $\bar{n}=1-n$.

It is useful ${ }^{16}$ to distinguish two cases: $n \geq \frac{1}{2}$ and $n<\frac{1}{2}$.

\section{B.2.1 Case $n \geq \frac{1}{2}$}

Here, we introduce the proposal $\pi_{*}^{1} \in \Pi$ defined as follows:

$$
g\left(\pi_{*}^{1}\right)=0 \text { and } s_{j}\left(\pi_{*}^{1}\right)=0 \text { for all } j \in[0,1]
$$

In the result below, we prove that $\pi_{*}^{1}$ is always $A S_{l}$ 's best response whenever $A S_{w}$ suggests zero subsidies to a set of losers of at least measure $\frac{1}{2}$.

\section{Lemma 2}

If $n \geq \frac{1}{2}$, then $R\left(\pi^{0}\right)=\pi_{*}^{1}$.

\footnotetext{
${ }^{15}$ We omit the dependence on $\pi^{0}$.

${ }^{16}$ Mathematically speaking, it is not necessary to consider the two cases. However, we introduce them here for the sake of completeness.
} 


\section{Proof:}

First, we show that $I\left(\pi^{0}, \pi_{*}^{1}\right)=1$. Indeed, for all $i \in N$,

$$
\sigma_{i}\left(g\left(\pi_{*}^{1}\right)\right)=g\left(\pi^{0}\right)\left(V_{l}-k\right)-\int_{0}^{1} s_{j}\left(\pi^{0}\right) d j \leq 0 .
$$

As a consequence, $u_{i}\left(\pi_{*}^{1}\right) \geq u_{i}\left(\pi^{0}\right)$ for all $i \in N$, and these individuals vote for $\pi_{*}^{1}$ because of Tie-Breaking Rule 1 . Since $n \geq \frac{1}{2}$ and due to Tie-Breaking Rule 2, proposal $\pi_{*}^{1}$ would be implemented against $\pi^{0}$, that is $I\left(\pi^{0}, \pi_{*}^{1}\right)=1$.

Second, observe that, for any arbitrary $\pi \in \Pi$,

$$
u_{1}(\pi)=g(\pi)\left(V_{l}-k\right)-\int_{0}^{1} s_{j}(\pi) d j \leq 0=u_{1}\left(\pi_{*}^{1}\right) .
$$

So $\pi_{*}^{1}$ yields the highest utility $A S_{l}$ can obtain under the Agenda-Setter Rule within the set of possible choices $\pi \in \Pi$. Moreover, notice that, if either $g(\pi)=1$ or $\int_{0}^{1} s_{j}(\pi) d j>0$, the inequality in (6) is strict and, hence, up to changes in sets of zero measure, $\pi_{*}^{1}$ is the unique best response when $n \geq \frac{1}{2}$.

In words, Lemma 2 makes it clear that the public project will never be adopted if $A S_{w}$ proposes $\pi^{0}$ such that $n \geq \frac{1}{2}$.

\section{B.2.2 Case $\mathrm{n}<\frac{1}{2}$}

First we split the set $\bar{N}$ into further subsets. For that purpose we define ${ }^{17}$

$$
\begin{aligned}
& \text { - } \Sigma_{0}:=\arg \min _{Q \subseteq \bar{N}}\left\{\int_{Q} \sigma_{j}(0) d j \mid q=\frac{1}{2}-n\right\}, \\
& \text { - } \Sigma_{1}:=\arg \min _{Q \subseteq \bar{N}}\left\{\int_{Q} \sigma_{j}(1) d j \mid q=\frac{1}{2}-n\right\} .
\end{aligned}
$$

where $\sigma_{j}(0)$ and $\sigma_{j}(1)$, for all $j \in[0,1]$, have been defined in (4) for proposals $\pi^{0}$ and $\pi^{1}$ such that $g\left(\pi^{1}\right)=0$ and $g\left(\pi^{1}\right)=1$ respectively. ${ }^{18}$ In words, $\Sigma_{0}$ is composed of

\footnotetext{
${ }^{17}$ Note that, given $\pi^{0}, \pi^{1} \in \Pi$, the function $f:[0,1] \rightarrow \mathbb{R}_{+}$defined by $f(i)=\sigma_{i}\left(g\left(\pi^{1}\right)\right)$ is Lebesguemeasurable and Lebesgue-integrable since $s\left(\pi^{0}\right)$ is Lebesgue-measurable and Lebesgue-integrable. Rigorously speaking, for the definition of $\Sigma_{0}$ and $\Sigma_{1}$ the minimization must be taken over a maximal compact set of sets $Q \subseteq \bar{N}$, so that both minimization problems have a solution, i.e., $\Sigma_{0}, \Sigma_{1} \neq \emptyset$.

${ }^{18}$ Notice that, for each $Q_{0} \in \Sigma_{0}$ and $Q_{1} \in \Sigma_{0}$, it holds that $q_{0}=q_{1}=\frac{1}{2}-n$, and that $A S_{w}$ and $A S_{l}$ do not belong to $Q_{0} \cup Q_{1}$ since, by definition, they do not belong to $\bar{N}$.
} 
all sets $Q_{0} \subseteq \bar{N}$ of measure $\frac{1}{2}-n$ containing individuals $j$ with smallest aggregate $\sigma_{j}(0)$. Similarly, $\Sigma_{1}$ is composed of all sets $Q_{1} \subseteq \bar{N}$ of measure $\frac{1}{2}-n$ containing individuals $j$ with smallest aggregate $\sigma_{j}(1)$. We point out that $Q_{0} \in \Sigma_{0}$ and $Q_{1} \in \Sigma_{1}$ independently of the exact design of the subsidy scheme $s\left(\pi^{1}\right)$ since, according to (4), the sign of $\sigma_{j}\left(g\left(\pi^{1}\right)\right)-\sigma_{i}\left(g\left(\pi^{1}\right)\right)$ for any $i, j \in[0,1]$ with $i \neq j$ depends only on $\pi^{0}$ and $g\left(\pi^{1}\right) \in\{0,1\}$. We also note that for some choices of $\pi^{0}$, different elements $Q_{0}, Q_{0}^{\prime} \in \Sigma_{0}$ (or $Q_{1}, Q_{1}^{\prime} \in \Sigma_{1}$ ) may differ even in a subset of individuals of non-zero measure. ${ }^{19}$ It turns out that outcomes of the game will be independent of a particular choice of $Q_{0}$ and $Q_{1}$. We select two arbitrary elements $Q_{0} \in \Sigma_{0}$ and $Q_{1} \in \Sigma_{1}$.

Second, we define two specific proposals. On the one hand, we define $\tilde{\pi}^{1} \in \Pi$, where

$$
g\left(\tilde{\pi}^{1}\right)=0, \quad s_{i}\left(\tilde{\pi}^{1}\right)= \begin{cases}\max \left\{0, \sigma_{i}(0)\right\} & \text { for } i \in Q_{0}, \\ 0 & \text { for all other } i .\end{cases}
$$

Notice that, according to $(4), \sigma_{i}\left(\tilde{\pi}^{1}\right)$ depends on $\int_{0}^{1} s_{j}\left(\sigma_{j}\left(\tilde{\pi}^{1}\right)\right) d j$ for all $i \in[0,1]$. That implies that $(7)$ defines $s_{i}\left(\tilde{\pi}^{1}\right)$ only implicitly. We next show that, in fact, $s_{i}\left(\tilde{\pi}^{1}\right)$ is well-defined for all $i \in[0,1]$.

\section{Remark 1}

The proposal $s\left(\tilde{\pi}^{1}\right)$ implicitly defined in (7) is well-defined.

\section{Proof:}

Given $\pi^{0}$ and $g\left(\pi^{1}\right)$, let $b_{i}:=s_{i}\left(\pi^{0}\right)+\left(g\left(\pi^{0}\right)-g\left(\pi^{1}\right)\right)\left(v_{i}-k\right)-\int_{0}^{1} s_{j}\left(\pi^{0}\right) d j$ for all $i \in[0,1]$. By $(7)$,

$$
\int_{0}^{1} s_{j}\left(\tilde{\pi}^{1}\right) d j=\int_{Q_{0}} \max \left\{0, b_{j}+\int_{0}^{1} s_{j}\left(\tilde{\pi}^{1}\right) d j\right\} d j .
$$

Rearranging terms, we obtain

$$
\int_{0}^{1} s_{j}\left(\tilde{\pi}^{1}\right) d j=\frac{1}{1-\left|Q_{0}^{\int_{0}^{1} s_{j}\left(\tilde{\pi}^{1}\right) d j}\right|} \cdot \int_{Q_{0}^{\int_{0}^{1} s_{j}\left(\tilde{\pi}^{1}\right) d j}} b_{j} d j
$$

where, for any $x \in \mathbb{R}_{+}$, we define $Q_{0}^{x}:=\left\{j \in Q_{0} \mid b_{j}+x \geq 0\right\}$. If we additionally define

$$
f(x):=x-\frac{1}{1-\left|Q_{0}^{x}\right|} \int_{Q_{0}^{x}} b_{j} d j
$$

\footnotetext{
${ }^{19}$ For instance, if $s_{i}\left(\pi^{0}\right)=s>s_{j}\left(\pi^{0}\right)$ for all $i \in A$ and all $j \notin A$, with $s>0$ and $A \subseteq[0,1]$ a measurable set of measure larger than $\frac{1}{2}$, then $\sigma_{i}\left(g\left(\pi^{1}\right)\right)$ is the same for all $i \in A$, so any subset of $A$ of measure $\frac{1}{2}$ belongs to $\Sigma_{g\left(\pi^{1}\right)}$.
} 
the existence of a solution $s\left(\tilde{\pi}^{1}\right)$ to equation (8) such that $\int_{0}^{1} s_{j}\left(\tilde{\pi}^{1}\right) d j=x$ is equivalent to the existence of a solution $x$ to the equation

$$
f(x)=0 .
$$

Under the assumptions of the paper, $f(\cdot)$ is a continuous function. Moreover, $f(0) \leq 0$ and $\lim _{x \rightarrow+\infty} f(x)=+\infty$. As a consequence, equation (9) has a (maybe not unique) solution. If there is only one solution $x$ to equation (9), we choose $s\left(\tilde{\pi}^{1}\right)$ as the unique solution to equation (8). If there are multiple solutions to equation (9), we choose $s\left(\tilde{\pi}^{1}\right)$ as the unique solution to equation (8) such that $\int_{0}^{1} s_{j}\left(\tilde{\pi}^{1}\right) d j=x^{*}$, where $x^{*}$ is the minimum ${ }^{20}$ solution to equation (9). The reason for this latter choice is that - ceteris paribus - the second agenda-setter will always prefer smaller aggregate subsidies. As a consequence, $s\left(\tilde{\pi}^{1}\right)$ is uniquely defined up to a relabeling of agents and changes in sets of measure zero.

On the other hand, we define $\tilde{\tilde{\pi}}^{1} \in \Pi$, where

$$
g\left(\tilde{\tilde{\pi}}^{1}\right)=1, \quad s_{i}\left(\tilde{\tilde{\pi}}^{1}\right)= \begin{cases}\max \left\{0, \sigma_{i}(1)\right\} & \text { for } i \in Q_{1}, \\ 0 & \text { for all other } i .\end{cases}
$$

We note that, like $\tilde{\pi}^{1}, \tilde{\tilde{\pi}}^{1}$ is well-defined.

Next we prove that we can present the reaction function of $A S_{l}$ in a simple way. ${ }^{21}$

\section{Lemma 3}

If $n<\frac{1}{2}$, then

$$
R\left(\pi^{0}\right)= \begin{cases}\tilde{\pi}^{1} & \text { if } u_{1}\left(\tilde{\tilde{\pi}}^{1}\right) \geq u_{1}\left(\tilde{\pi}^{1}\right) \\ \tilde{\pi}^{1} & \text { otherwise. }\end{cases}
$$

\section{Proof:}

We first prove that $s_{i}\left(R\left(\pi^{0}\right)\right)=0$ for all $i \in N$. Indeed, since $s_{i}\left(\pi^{0}\right)=0$ for all $i \in N$, we obtain

$$
\begin{aligned}
\sigma_{i}\left(g\left(R\left(\pi^{0}\right)\right)\right) & =\left[g\left(\pi^{0}\right)-g\left(R\left(\pi^{0}\right)\right)\right]\left(V_{l}-k\right)-\left(\int_{0}^{1} s_{j}\left(\pi^{0}\right) d j-\int_{0}^{1} s_{j}\left(R\left(\pi^{0}\right)\right) d j\right) \\
& =u_{1}\left(\pi^{0}\right)-u_{1}\left(R\left(\pi^{0}\right)\right) \leq 0
\end{aligned}
$$

\footnotetext{
${ }^{20}$ By the above reasoning it is obvious that $x^{*}$ exists.

${ }^{21}$ As we prove in Lemma $3, R(\cdot)$ has just one element up to a relabeling of individuals and changes in sets of Lebesgue-measure zero. Hence, the best response for $A S_{l}$ is described by a function rather than a correspondence.
} 
for all $i \in N$, where the inequality holds by definition of best response.

Second, we show that $s_{i}\left(R\left(\pi^{0}\right)\right)=\max \left\{0, \sigma_{i}\left(g\left(R\left(\pi^{0}\right)\right)\right)\right\}$ for all $i \in Q_{g\left(R\left(\pi^{0}\right)\right)}$, where $g\left(R\left(\pi^{0}\right)\right) \in\{0,1\}$. Indeed, since $u_{1}\left(\pi^{1}\right)$ is decreasing as a function of aggregate subsidies of the counter-proposal $\pi^{1}, A S_{l}$ has a strict incentive to form the "cheapest" majority that ensures $I\left(\pi^{0}, \pi^{1}\right)=1$. Thus, due to Tie-breaking Rule 2 , she only pays subsidies to half of the electorate, and she pays them the minimal amount that will prompt them to vote for $R\left(\pi^{0}\right)$. Because any $A S_{l}$ 's best response will secure the votes of individuals in $N$, she needs the additional support of a fraction of $\frac{1}{2}-n$ individuals. The tax burden is smallest if those individuals in $\bar{N}$ for which $\sigma_{j}\left(g\left(R\left(\pi^{0}\right)\right)\right)$ is smallest, i.e., $Q_{g\left(R\left(\pi^{0}\right)\right)}$, are subsidized. Moreover, it cannot be profitable to pay higher subsidies than $\max \left\{0, \sigma_{i}\left(g\left(R\left(\pi^{0}\right)\right)\right)\right\}$ to each individual $i \in Q_{g\left(R\left(\pi^{0}\right)\right)}$. Note that depending on whether $g\left(R\left(\pi^{0}\right)\right)=0$ or $g\left(R\left(\pi^{0}\right)\right)=1$, the chosen coalitions may differ, so $Q_{0}$ is in general different from $Q_{1} \cdot{ }^{22}$

Third, we observe that, since the above subsidy scheme already guarantees the support of half of the society for $R\left(\pi^{0}\right), A S_{l}$ will not pay further subsidies, that is $s_{i}\left(R\left(\pi^{0}\right)\right)=0$ for all other individuals $i$. Therefore the aggregate amount of subsidies under $R\left(\pi^{0}\right)$ is

$$
\int_{0}^{1} s_{j}\left(R\left(\pi^{0}\right)\right) d j=\int_{Q_{g\left(R\left(\pi^{0}\right)\right)}} \max \left\{0, \sigma_{j}\left(g\left(R\left(\pi^{0}\right)\right)\right)\right\} d j .
$$

Fourth, given the subsidy scheme $s\left(R\left(\pi^{0}\right)\right)$, only one alternative for the best response, $R\left(\pi^{0}\right)$, remains. Either it is optimal for $A S_{l}$ to choose $g\left(R\left(\pi^{0}\right)\right)=0$ or it is optimal to choose $g\left(R\left(\pi^{0}\right)\right)=1$. That is, in each respective case either $\tilde{\pi}^{1}$ is a best response or $\tilde{\pi}^{1}$ is.

Lastly, we note that $g\left(R\left(\pi^{0}\right)\right)=1$ holds if and only if $u_{1}\left(\tilde{\tilde{\pi}}^{1}\right) \geq u_{1}\left(\tilde{\pi}^{1}\right)$ as

$$
u_{1}\left(\pi^{0}\right)=u_{j}\left(\pi^{0}\right), u_{1}\left(\tilde{\pi}^{1}\right)=u_{j}\left(\tilde{\pi}^{1}\right) \text { and } u_{1}\left(\tilde{\tilde{\pi}}^{1}\right)=u_{j}\left(\tilde{\tilde{\pi}}^{1}\right) \text { for all } j \in N,
$$

and

$$
\max \left\{u_{1}\left(\tilde{\pi}^{1}\right), u_{1}\left(\tilde{\tilde{\pi}}^{1}\right)\right\} \geq u_{1}\left(\pi^{0}\right)
$$

and Tie-Breaking Rule 3 applies. Expression (11) is straightforward, whereas (12) is a consequence of Lemma 1 and the fact that $\tilde{\pi}^{1}$ (resp. $\tilde{\tilde{\pi}}^{1}$ ) is the best-response if

\footnotetext{
${ }^{22}$ The argument in this paragraph depends on whether such a subsidy scheme is well-defined. This question was answered in the affirmative in Remark 1.
} 
$g\left(R\left(\pi^{0}\right)\right)=0$ (resp. $\left.g\left(R\left(\pi^{0}\right)\right)=1\right)$. We further note that (11) shows that the interest of $A S_{l}$ coincides exactly with the interests of voters in $N$ regarding the choice between $\tilde{\pi}^{1}, \tilde{\tilde{\pi}}^{1}$, and $\pi^{0}$.

\section{B.3 The optimal strategy of the first agenda-setter}

Once the best response of the second agenda-setter has been analyzed, we use backward induction to study the optimal strategy for the first agenda-setter, $A S_{w}$. For the analysis it is useful to introduce the parameter $\hat{p} \in(0,1)$, where

$$
\hat{p}=\frac{k-V_{l}}{V_{w}-V_{l}} .
$$

The existence and uniqueness of $\hat{p} \in(0,1)$ is guaranteed, since $V_{w}-k>0, V_{l}-k<0$ and $p V_{w}+(1-p) V_{l}-k$ is linear in $p$. We note that projects are socially efficient if and only if $p \geq \hat{p}$.

In the following, we introduce two specific proposals that will turn out to be optimal proposals for the first agenda-setter in some particular circumstances. On the one hand, for $p \geq \hat{p}$, consider the proposal $\pi_{*}^{0}$ defined as follows: ${ }^{23}$

$$
g\left(\pi_{*}^{0}\right)=1 \text { and } \quad s_{j}\left(\pi_{*}^{0}\right)= \begin{cases}\frac{\left(\frac{1}{2}-p+\hat{p}\right)\left[V_{w}-V_{l}\right]}{1-\left(\frac{1}{2}+\hat{p}\right)} & \text { for } j \in(0, p] \\ \frac{(1-p)\left[V_{w}-V_{l}\right]}{1-\left(\frac{1}{2}+\hat{p}\right)} & \text { for } j \in\left(p, \frac{1}{2}+\hat{p}\right] \\ 0 & \text { for all other } j .\end{cases}
$$

Observe that, in order for $\pi_{*}^{0}$ to be well-defined, it is sufficient that $\frac{1}{2}-p+\hat{p} \geq 0$ and $1-\left(\frac{1}{2}+\hat{p}\right)>0$. Since $0<p<\frac{1}{2}$, it follows that both conditions hold if $\hat{p} \leq p$. On the other hand, let $\pi_{* *}^{0} \in \Pi$ be any proposal that satisfies $n \geq \frac{1}{2}$. It will transpire that the details of such a proposal do not matter.

The following lemma proves that the optimal strategy for the first agenda-setter, $A S_{w}$, depends solely on whether $p$ is larger or smaller than $\hat{p}$.

\footnotetext{
${ }^{23}$ We assume that $\hat{s}$ is sufficiently large for $s_{j}\left(\pi_{*}^{0}\right)$ as defined in (13) not to violate the upper bound on subsidies.
} 


\section{Lemma 4}

For $A S_{w}$ it is optimal to choose

$$
\pi^{0}= \begin{cases}\pi_{* *}^{0} & \text { if } p<\hat{p} \\ \pi_{*}^{0} & \text { if } p \geq \hat{p}\end{cases}
$$

\section{Proof:}

In the first stage, $A S_{w}$ chooses $\pi^{0} \in \boldsymbol{\pi}_{*}^{0}$, where

$$
\boldsymbol{\pi}_{*}^{0}=\underset{\pi^{0} \in \Pi}{\operatorname{argmax}}\left\{u_{0}\left(R\left(\pi^{0}\right)\right)=g\left(R\left(\pi^{0}\right)\right)\left(V_{w}-k\right)-\int_{0}^{1} s_{j}\left(R\left(\pi^{0}\right)\right) d j\right\} .
$$

We distinguish two cases depending on whether $\hat{p}$ is larger than $p$ or not.

Case 1: $p<\hat{p}$

We claim (and prove below) that if $n<\frac{1}{2}$, regardless of the exact strategy chosen by $A S_{w}, A S_{l}$ 's best response is $\tilde{\pi}^{1}$ and not $\tilde{\tilde{\pi}}^{1}$, as defined in (7) and (10) respectively. According to Lemma 2, whenever $n \geq \frac{1}{2}$ the best response for $A S_{l}$ is $\pi_{*}^{1}$, as defined in (5). As a consequence, $A S_{w}$ faces the following choice:

$$
u_{0}\left(R\left(\pi^{0}\right)\right)= \begin{cases}u_{0}\left(\pi_{*}^{1}\right)=0 & \text { if } n \geq \frac{1}{2} \\ u_{0}\left(\tilde{\pi}^{1}\right)=-\int_{0}^{1} s_{j}\left(\tilde{\pi}^{1}\right) d j \leq 0 & \text { if } n<\frac{1}{2}\end{cases}
$$

From (14) it follows that $A S_{w}$ will select any proposal $\pi^{0}$ such that $n \geq \frac{1}{2}$, i.e., $\pi_{* *}^{0}$.

It only remains to prove the above claim. We assume that

$$
u_{i}\left(\tilde{\tilde{\pi}}^{1}\right) \geq u_{i}\left(\pi^{0}\right)
$$

for all $i \in Q_{1}$. We note that if (15) does not hold for a subset of non-zero measure of $Q_{1}$, it must necessarily be the case that $I\left(\pi^{0}, \tilde{\tilde{\pi}}^{1}\right)=0$, due to the way $\tilde{\tilde{\pi}}^{1}$ is constructed. In that case, by definition of best response, $\tilde{\pi}^{1}$ cannot be the best response, and so, by Lemma $3 A S_{l}$ 's best response is $\tilde{\pi}^{1}$, as we claimed.

Let $\pi^{0} \in \Pi$ be an arbitrary strategy chosen by $A S_{w}$ so that $n<\frac{1}{2}$. First, we split set $Q_{1}$ into two disjoint subsets $Q_{1}^{w}$ and $Q_{1}^{l}$, where

$$
\begin{aligned}
& Q_{1}^{w}:=\left\{j \in Q_{1} \mid j \in(0, p]\right\}, \\
& Q_{1}^{l}:=\left\{j \in Q_{1} \mid j \in(p, 1)\right\} .
\end{aligned}
$$


Set $Q_{1}^{w}$ comprises all winners from $Q_{1}$ and set $Q_{1}^{l}$ all losers from $Q_{1}$. As $Q_{1}=Q_{1}^{w} \cup Q_{1}^{l}$ and $Q_{1}^{w} \cap Q_{1}^{l}=\emptyset$, we obtain $q_{1}^{w}+q_{1}^{l}=q_{1}=\frac{1}{2}-n$. Observe that, by definition, $q_{1}^{w} \leq p$ and hence, by assumption,

$$
q_{1}^{w}<\hat{p}
$$

Second, starting from $\tilde{\tilde{\pi}}^{1}$ it is helpful to define an auxiliary proposal ${ }^{24} \tilde{\pi}^{\prime 1} \in \Pi$ to be considered for the second agenda-setter, $A S_{l}$. Specifically, let $\tilde{\pi}^{\prime 1} \in \Pi$ be a proposal that comprises $g\left(\tilde{\pi}^{\prime 1}\right)=0$ and the following subsidy scheme:

$$
s_{j}\left(\tilde{\pi}^{\prime 1}\right)= \begin{cases}\max \left\{0, \sigma_{j}(1)\right\}+\frac{V_{w}-k}{1-q_{1}^{w}} & \text { for } j \in Q_{1}^{w}, \\ \max \left\{0, \sigma_{j}(1)\right\} & \text { for } j \in Q_{1}^{l}, \\ 0 & \text { for all other } j .\end{cases}
$$

The aggregate amount of subsidies under proposal $\tilde{\pi}^{\prime 1}$ is

$$
\int_{0}^{1} s_{j}\left(\tilde{\pi}^{\prime 1}\right) d j=\int_{j \in Q_{1}} \max \left\{0, \sigma_{j}(1)\right\} d j+q_{1}^{w} \frac{V_{w}-k}{1-q_{1}^{w}} .
$$

Third, we show that $u_{i}\left(\tilde{\pi}^{\prime 1}\right) \geq u_{i}\left(\pi^{0}\right)$ for all $i \in Q_{1}$, so by Tie-Breaking Rules 1 and 2 , $\tilde{\pi}^{\prime 1}$ would win a vote against $\pi^{0}$. On the one hand, consider $i \in Q_{1}^{w}$. Then

$$
\begin{aligned}
u_{i}\left(\tilde{\pi}^{\prime 1}\right) & =\max \left\{0, \sigma_{i}(1)\right\}+\frac{V_{w}-k}{1-q_{1}^{w}}-\int_{j \in Q_{1}} \max \left\{0, \sigma_{j}(1)\right\} d j-q_{1}^{w} \frac{V_{w}-k}{1-q_{1}^{w}} \\
& =V_{w}-k+\max \left\{0, \sigma_{i}(1)\right\}-\int_{j \in Q_{1}} \max \left\{0, \sigma_{j}(1)\right\} d j=u_{i}\left(\tilde{\tilde{\pi}}^{1}\right) \geq u_{i}\left(\pi^{0}\right),
\end{aligned}
$$

where the last inequality holds by (15) since $i \in Q_{1}$. On the other hand, consider $i \in Q_{1}^{l}$. Then

$$
\begin{aligned}
u_{i}\left(\tilde{\pi}^{\prime 1}\right) & =\max \left\{0, \sigma_{i}(1)\right\}-\int_{j \in Q_{1}} \max \left\{0, \sigma_{j}(1)\right\} d j-q_{1}^{w} \frac{V_{w}-k}{1-q_{1}^{w}} \\
& >V_{l}-k+\max \left\{0, \sigma_{i}(1)\right\}-\int_{j \in Q_{1}} \max \left\{0, \sigma_{j}(1)\right\} d j=u_{i}\left(\tilde{\tilde{\pi}}^{1}\right) \geq u_{i}\left(\pi^{0}\right),
\end{aligned}
$$

where the last inequality again holds by (15) since $i \in Q_{1}$ and the strict inequality holds using the definition of $\hat{p}$ since (16) implies that

$$
q_{1}^{w} \frac{V_{w}-k}{1-q_{1}^{w}}<k-V_{l}
$$

\footnotetext{
${ }^{24}$ Notice that $s\left(\tilde{\pi}^{1}\right)$ is again defined only implicitly. However, the fact that this proposal is welldefined follows from a similar argument as for proposals $\tilde{\pi}^{1}$ and $\tilde{\tilde{\pi}}^{1}$ defined in (7) and (10) respectively (see Remark 1).
} 
Fourth, $A S_{l}$ will prefer the implementation of $\tilde{\pi}^{1}$ to $\tilde{\tilde{\pi}}^{1}$. Indeed,

$$
\begin{aligned}
u_{1}\left(\tilde{\pi}^{\prime 1}\right) & =-q_{1}^{w} \frac{V_{w}-k}{1-q_{1}^{w}}-\int_{j \in Q_{1}} \max \left\{0, \sigma_{j}(1)\right\} d j \\
& >\left(V_{l}-k\right)-\int_{j \in Q_{1}} \max \left\{0, \sigma_{j}(1)\right\} d j=u_{1}\left(\tilde{\tilde{\pi}}^{1}\right),
\end{aligned}
$$

where the inequality holds by (16).

Finally, since $\tilde{\pi}^{1}$ is the optimal response for $A S_{l}$ provided that the project is not implemented and $A S_{l}$ prefers to implement $\tilde{\pi}^{1}$ to $\tilde{\tilde{\pi}}^{1}$, it must be the case that $\tilde{\pi}^{1}$ wins a vote against $\pi^{0}$ and that $u_{1}\left(\tilde{\pi}^{1}\right) \geq u_{1}\left(\tilde{\pi}^{\prime 1}\right)>u_{1}\left(\tilde{\tilde{\pi}}^{1}\right)$. Therefore it is optimal for $A S_{l}$ to choose $\tilde{\pi}^{1}$, as we claimed.

Case 2: $p \geq \hat{p}$

We show that proposal $\pi_{*}^{0}$ introduced in (13), which is well-defined if $p \geq \hat{p}$, induces $g\left(R\left(\pi_{*}^{0}\right)\right)=1$ and $s_{j}\left(R\left(\pi_{*}^{0}\right)\right)=0$ for all $j \in[0,1]$. Indeed, it is not difficult to check that $\pi_{*}^{0}$ yields

$$
t\left(\pi_{*}^{0}\right)=k+\frac{\left(\frac{1}{2}-p+\hat{p}\right)\left[V_{w}-V_{l}\right]}{1-\left(\frac{1}{2}+\hat{p}\right)}
$$

and

$$
u_{j}\left(\pi_{*}^{0}\right)=V_{w}-k \text { for all } j \in\left(0, \frac{1}{2}+\hat{p}\right] .
$$

Moreover, $N=\left(\frac{1}{2}+\hat{p}, 1\right)$, which implies $n=\frac{1}{2}-\hat{p}$. Since $\hat{p}>0$, then $n<\frac{1}{2}$, so both $Q_{0}$ and $Q_{1}$ are sets of positive measure. By Lemma 3 we know that $A S_{l}$ will choose $\tilde{\pi}^{1}$ if $u_{1}\left(\tilde{\tilde{\pi}}^{1}\right) \geq u_{1}\left(\tilde{\pi}^{1}\right)$ and will choose $\tilde{\pi}^{1}$ otherwise.

On the one hand, proposal $\tilde{\pi}^{1}$ is given $\operatorname{by}^{25} g\left(\tilde{\pi}^{1}\right)=0$ and

$$
s_{j}\left(\tilde{\pi}^{1}\right)= \begin{cases}0 & \text { for } j \in\left[0, \frac{1}{2}\right) \cup\left(\frac{1}{2}+\hat{p}, 1\right], \\ V_{w}-V_{l} & \text { for } j \in Q_{0}=\left[\frac{1}{2}, \frac{1}{2}+\hat{p}\right] .\end{cases}
$$

Therefore $^{26} u_{1}\left(\tilde{\pi}^{1}\right)=-\hat{p} \frac{V_{w}-k}{1-\hat{p}}=-\hat{p}\left(V_{w}-V_{l}\right)$.

On the other hand, it is also easy to check that proposal $\tilde{\tilde{\pi}}^{1}$ is given by $g\left(\tilde{\tilde{\pi}}^{1}\right)=1$ and $s_{j}\left(\tilde{\tilde{\pi}}^{1}\right)=0$ for all $j \in[0,1]$. Thus,

$$
u_{1}\left(\tilde{\tilde{\pi}}^{1}\right)=V_{l}-k=-\hat{p}\left(V_{w}-V_{l}\right)=u_{1}\left(\tilde{\pi}^{1}\right),
$$

\footnotetext{
${ }^{25}$ Note again that there are other possible choices of $\tilde{\pi}^{1}$ and $Q_{0}$, as long as $q_{0}=\hat{p}$ and $Q_{0} \subseteq$ $\left(0, \frac{1}{2}+\hat{p}\right)$. However, the equilibrium outcome will not depend on the specific choice.

${ }^{26}$ Subsidies in $\tilde{\pi}^{1}$ are bounded from above by $\hat{s}$ (for sufficiently high $\hat{s}$ ) and are non-negative because $\hat{p}<1$.
} 
where the second equality holds by the definition of $\hat{p}$.

Since for $A S_{l}$ proposal $\tilde{\pi}^{1}$ yields the same utility as proposal $\tilde{\pi}^{1}$, by Tie-Breaking Rule 3 the equilibrium proposal is $\tilde{\tilde{\pi}}^{1}$. Thus $g\left(R\left(\pi_{*}^{0}\right)\right)=1$, as claimed.

Lastly, for any $\pi \in \Pi$ such that either $g(\pi)=0$ or $\int_{0}^{1} s_{j}(\pi) d j>0$ we have

$$
u_{0}\left(R\left(\pi_{*}^{0}\right)\right)=V_{w}-k>u_{0}(\pi) .
$$

As a consequence, it is optimal for $A S_{w}$ to choose $\pi_{*}^{0}$.

The preceding results (Lemmas 2, 3, and 4) establish Theorem 1. On the one hand, when $p<\hat{p}$ the first agenda-setter, $A S_{w}$, will propose $\pi_{* *}^{0}$, the second agenda-setter will counter-propose $\pi_{*}^{0}$, and the latter proposal will win the voting and be implemented. Since $\pi_{*}^{0}$ prescribes no public project implementation and no subsidies, it is the socially efficient solution. On the other hand, when $p \geq \hat{p}$ the first agenda-setter, $A S_{w}$, will choose $\pi_{*}^{0}$ and then $A S_{l}$ will respond with a proposal that prescribes public project implementation coupled with no subsidies, which constitutes the socially efficient solution. 


\section{Appendix C}

Here we prove Propositions 2, 3, 4, and 5.

\section{C.1 When amendments are not allowed}

We consider a one-round institution defined as follows: There is only one agenda-setter, a winner denoted by $A S_{w}$. She makes a proposal $\pi^{0}$ which is either accepted or rejected by the citizens. In the latter case, the status quo prevails, yielding zero utility for all individuals. The decision is taken according to the simple majority rule. The utility functions of all individuals are exactly the same as in the rest of the paper. Likewise, taxes are uniform, and subsidies to all individuals other than the agenda-setter are only constrained to be non-negative and bounded from above. The Agenda-Setter Rule prevails and is now only applied to $A S_{w}$. To examine one-round procedures, Tie-Breaking Rules in Section 3 are modified as follows:

\section{Tie-Breaking Rule 4}

If $u_{i}\left(\pi^{0}\right)=0$, individual $i$ will vote for the proposal $\pi^{0}$.

\section{Tie-Breaking Rule 5}

If one half of the society votes in favor of $\pi^{0}$ and the other half in favor of the status quo, the proposal $\pi^{0}$ will be implemented.

\section{Tie-Breaking Rule 6}

If $A S_{w}$ is indifferent between a proposal $\pi^{0} \in \Pi$ involving $g\left(\pi^{0}\right)=1$ and a proposal $\pi^{\prime 0}$ involving $g\left(\pi^{\prime 0}\right)=0$, she will always suggest $\pi^{0}$.

As only one proposal is made, the Tie-Breaking Rules favor this proposal over the status quo. To show how particular inefficiencies occur, we considerer parameter constellations that fulfill

$$
V_{w} \geq V_{w}^{*}:=\frac{k-\left(\frac{1}{2}-p\right) V_{l}}{1-\left(\frac{1}{2}-p\right)} .
$$

This assumption is equivalent to $\hat{p} \leq p+\frac{1}{2}$. We also assume that $p<\hat{p}$, i.e., it is not socially efficient to implement the project. In the proof of Proposition 2 below we show that, under the above assumptions, it is always optimal for $A S_{w}$ to propose to implement the public project and get her proposal approved. Moreover, the opti- 
mal proposal for the agenda-setter includes strictly positive subsidies. Consequently, inefficiencies of type 1 and 3 arise.

\section{Proof of Proposition 2:}

The agenda-setter $A S_{w}$ chooses a proposal according to

$$
\max _{\pi^{0} \in \Pi}\left\{u_{0}\left(\pi^{0}\right)=g\left(\pi^{0}\right)\left(V_{w}-k\right)-\int_{0}^{1} s_{j}\left(\pi^{0}\right) d j\right\}
$$

Let $\pi_{*}^{0} \in \Pi$ be such that $g\left(\pi_{*}^{0}\right)=0$ and $s_{j}\left(\pi_{*}^{0}\right)=0$ for all $j \in[0,1]$. Since $u_{0}\left(\pi_{*}^{0}\right)=0=$ $u_{j}\left(\pi_{*}^{0}\right)$ for all $j \in[0,1]$, by Tie-Breaking Rule $4 \pi_{*}^{0}$ would be unanimously supported. As the agenda-setter cannot obtain subsidies, $\pi_{*}^{0}$ is her optimal proposal provided she does not want to propose the public project.

We next consider proposals that involve project adoption. We claim that $A S_{w}$ cannot do better than with $\pi_{* *}^{0}$, which is defined by $g\left(\pi_{* *}^{0}\right)=1$ and the following subsidy scheme:

$$
s_{j}\left(\pi_{* *}^{0}\right)= \begin{cases}V_{w}^{*}-V_{l} & \text { for } j \in\left(p, \frac{1}{2}\right] \\ 0 & \text { for all other } j\end{cases}
$$

Notice that $V_{w}^{*}-V_{l}>0$ because $k>V_{l}$. Thus, the above proposal is feasible, since it involves non-negative subsidies and is bounded from above by $\hat{s}$ for a sufficiently large $\hat{s}$. For all $j \in[0, p]$, we have

$$
u_{j}\left(\pi_{* *}^{0}\right)=V_{w}-k-\left(\frac{1}{2}-p\right)\left(V_{w}^{*}-V_{l}\right)=\left[\frac{1-\hat{p}}{\hat{p}}-\frac{1-\left(\frac{1}{2}+p\right)}{\left(\frac{1}{2}+p\right)}\right]\left(k-V_{l}\right) \geq 0,
$$

where the inequality holds since $\hat{p} \leq \frac{1}{2}+p$ and $f(x)=\frac{1-x}{x}$ is decreasing in $x \in(0,1]$. Moreover, for all $j \in\left(p, \frac{1}{2}\right]$

$$
u_{j}\left(\pi_{* *}^{0}\right)=V_{l}-k+V_{w}^{*}-V_{l}-\left(\frac{1}{2}-p\right)\left(V_{w}^{*}-V_{l}\right)=0
$$

whereas, for all $j \in\left(\frac{1}{2}, 1\right]$

$$
u_{j}\left(\pi_{* *}^{0}\right)=V_{l}-k-\left(\frac{1}{2}-p\right)\left(V_{w}^{*}-V_{l}\right)<0 .
$$

By Tie-Breaking Rules 4 and 5, all individuals $j \in\left[0, \frac{1}{2}\right]$ will vote for $\pi_{* *}^{0}$, and the proposal will be carried out. 
Lastly, by (20), under $\pi_{* *}^{0}$, the utility of the agenda-setter $A S_{w}$ is non-negative. Moreover, a smaller (and non-negative) level of subsidies would not gain the support of $50 \%$ of the citizens. Therefore among the proposals that involve project adoption $A S_{w}$ 's optimal proposal is $\pi_{* *}^{0}$. Finally, due to Tie-Breaking Rule 6, we have $\pi_{* *}^{0}$ proposed and implemented. Hence, inefficiencies of type 3 will occur. As the project is socially inefficient, inefficiencies of type 1 will also occur.

Next, we consider the case where subsidy schemes are restricted to belonging to some given $\mathcal{A} \subseteq \mathbb{S}$ with $\mathcal{A} \neq \emptyset$. As before, individual subsidies are also bounded from above by $\hat{s}$. We further assume that, if $s(\pi)$ and $s^{\prime}(\pi)$ differ only on a set of measure zero, then $s(\pi) \in \mathcal{A}$ if and only if $s^{\prime}(\pi) \in \mathcal{A}$. $^{27}$

\section{Proof of Proposition 3:}

Let $\pi_{* *}^{0}$ be the proposal defined in the proof of Proposition 2 and let $V_{w}^{*}$ be as defined in (19). Assume that $\hat{p} \leq \frac{1}{2}+p$ or, equivalently, $V_{w} \geq V_{w}^{*}$. We claim that, up to changes on sets of measure zero and a relabeling of losers, a necessary condition for $A S_{w}$ to propose $\pi^{0} \in \Pi$ such that $g\left(\pi^{0}\right)=1$ is

$$
s_{j}\left(\pi^{0}\right) \geq s_{j}\left(\pi_{* *}^{0}\right) \text { for all } j \in[0,1] .
$$

Indeed, if $A S_{w}$ wants $\pi^{0}$ to be approved, due to Tie-Breaking Rules 4 and $5, \pi^{0}$ must give non-negative utility to at least half of the society. Hence, at least a fraction of $\frac{1}{2}-p$ losers must have non-negative utility under $\pi^{0}$. We can assume without loss of generality that these individuals are located in $H:=\left(p, \frac{1}{2}\right]$. Then,

$$
\begin{aligned}
0 \leq \int_{H} u_{i}\left(\pi^{0}\right) d i & =\int_{H}\left[V_{l}-k+s_{i}\left(\pi^{0}\right)-\int_{0}^{1} s_{j}\left(\pi^{0}\right) d j\right] d i \\
& =\left(\frac{1}{2}-p\right)\left(V_{l}-k\right)-\left(\frac{1}{2}-p\right) \int_{0}^{1} s_{j}\left(\pi^{0}\right) d j+\int_{H} s_{j}\left(\pi^{0}\right) d j \\
& \leq\left(\frac{1}{2}-p\right)\left(V_{l}-k\right)+\left(1-\left(\frac{1}{2}-p\right)\right) \int_{0}^{1} s_{j}\left(\pi^{0}\right) d j
\end{aligned}
$$

which implies

$$
\int_{0}^{1} s_{j}\left(\pi^{0}\right) d j \geq \frac{1-\left(\frac{1}{2}+p\right)}{\frac{1}{2}+p}\left(k-V_{l}\right)=\left(\frac{1}{2}-p\right)\left(V_{w}^{*}-V_{l}\right) .
$$

\footnotetext{
${ }^{27}$ This assumption is not necessary for the result, but it simplifies the proof.
} 
As a consequence, we have for all $j \in\left(p, \frac{1}{2}\right]$

$$
\begin{gathered}
0 \leq u_{j}\left(\pi^{0}\right) \leq\left(V_{l}-k\right)+s_{j}\left(\pi^{0}\right)-\frac{1-\left(\frac{1}{2}+p\right)}{\frac{1}{2}+p}\left(k-V_{l}\right) \\
=s_{j}\left(\pi^{0}\right)-\frac{\left(k-V_{l}\right)}{\frac{1}{2}+p}=s_{j}\left(\pi^{0}\right)-\left(V_{w}^{*}-V_{l}\right)
\end{gathered}
$$

so (22) holds. Hence we can write $s_{j}\left(\pi^{0}\right)=s_{j}\left(\pi_{* *}^{0}\right)+\delta_{j}$, where $\delta_{j} \geq 0$ for all $j \in[0,1]$. We claim that, up to changes on subsets of measure zero,

$$
\delta_{i} \geq \int_{0}^{1} \delta_{j} d j, \text { for all } i \in H
$$

Suppose otherwise, i.e., $\delta_{i}<\int_{0}^{1} \delta_{j} d j$ for all $i$ belonging to a subset of non-zero measure $B \subseteq H$. Then for all $i \in B$, we have

$$
\begin{aligned}
u_{i}\left(\pi^{0}\right) & =V_{l}-k+s_{i}\left(\pi^{0}\right)-\int_{0}^{1} s_{j}\left(\pi^{0}\right) d j=V_{l}-k+s_{i}\left(\pi_{* *}^{0}\right)+\delta_{i}-\int_{0}^{1} s_{j}\left(\pi_{* *}^{0}\right) d j-\int_{0}^{1} \delta_{j} d j \\
& <V_{l}-k+s_{i}\left(\pi_{* *}^{0}\right)-\int_{0}^{1} s_{j}\left(\pi_{* *}^{0}\right) d j=0,
\end{aligned}
$$

where the last equality holds by (21). This contradicts the fact that all voters $i \in B$ vote for $\pi^{0}$ and not for the status quo. Now let $i \in[0, p]$. Then, $u_{i}\left(\pi^{0}\right) \geq 0$ if and only if

$$
0 \leq V_{w}-k+s_{i}\left(\pi^{0}\right)-\int_{0}^{1} s_{j}\left(\pi^{0}\right) d j=\left[\frac{1-\hat{p}}{\hat{p}}-\frac{1-\left(\frac{1}{2}+p\right)}{\left(\frac{1}{2}+p\right)}\right]\left(k-V_{l}\right)+\delta_{i}-\int_{0}^{1} \delta_{j} d j,
$$

where the equality holds by (20). By the non-negativity of $\delta_{i}$ and the Agenda-Setter Rule - which implies $\delta_{0}=0$ - we obtain that $u_{i}\left(\pi^{0}\right) \geq 0$ for all $i \in[0, p]$ is equivalent to

$$
\int_{0}^{1} \delta_{j} d j \leq\left[\frac{V_{w}-k}{k-V_{l}}-\frac{1-\left(\frac{1}{2}+p\right)}{\left(\frac{1}{2}+p\right)}\right]\left(k-V_{l}\right) .
$$

In conclusion, for a proposal $\pi^{0}$ with $g\left(\pi^{0}\right)=1$ to be proposed by $A S_{w}$ and approved by at least half of the society, it is necessary that $s_{j}\left(\pi^{0}\right)=s_{j}\left(\pi_{* *}^{0}\right)+\delta_{j}$ for all $j \in[0,1]$, where (23) and (24) hold.

For a given project $\left(V_{w}, V_{l}, k, p\right) \in \mathcal{P}$, let $A\left(V_{w}, V_{l}, k, p\right) \subseteq \mathcal{A}$ be the (maybe empty) set of subsidy schemes that satisfy $(23)$ and $(24)$, and let $D\left(V_{w}, V_{l}, k, p\right)$ be the subset of $A\left(V_{w}, V_{l}, k, p\right)$ for which $\int_{0}^{1} \delta_{j} d j$ is minimum. Repeating exactly the same arguments 
as in the proof of Proposition 2, we find that the optimal choice for $A S_{w}$ is ${ }^{28}$

$$
\pi^{0}= \begin{cases}\pi \text { with } g(\pi)=1 \text { and } s(\pi) \in D\left(V_{w}, V_{l}, k, p\right) & \text { if } D\left(V_{w}, V_{l}, k, p\right) \neq \emptyset \\ \pi_{*}^{0} & \text { otherwise }\end{cases}
$$

where $\pi_{*}^{0}$ has been defined by $g\left(\pi_{*}^{0}\right)=0$ and $s_{j}\left(\pi_{*}^{0}\right)=0$ for all $j \in[0,1]$.

Finally, we can define two different projects $\left(V_{w}, V_{l}, k, p\right)$ and $\left(V_{w}^{\prime}, V_{l}^{\prime}, k^{\prime}, p^{\prime}\right)$ such that ${ }^{29}$

$$
p<\hat{p}<\frac{1}{2}+p, \quad \hat{p}^{\prime}<p^{\prime} \quad \text { and } \quad D=D\left(V_{w}, V_{l}, k, p\right)=D\left(V_{w}^{\prime}, V_{l}^{\prime}, k^{\prime}, p^{\prime}\right) .
$$

On the one hand, if $D \neq \emptyset$, then project $\left(V_{w}, V_{l}, k, p\right)$ will be implemented, although it is not socially efficient. Moreover, aggregate subsidies are strictly positive. On the other hand, if $D=\emptyset$, then project $\left(V_{w}^{\prime}, V_{l}^{\prime}, k^{\prime}, p^{\prime}\right)$ will not be undertaken, although its implementation is socially efficient. In the first case, inefficiencies of type 1 and 3 arise, whereas inefficiencies of type 2 arise in the second case.

\section{C.2 When the political initiative is given to losers}

We consider the same two-round institution studied in Section 5, with the modification that $A S_{l}$ is the first agenda-setter (she proposes $\pi^{1}$ ) and $A S_{w}$ is the second agenda-setter (she counter-proposes $\pi^{0}$ ). We modify Tie-Breaking Rules 1-3 so that the amendment wins in case of indifferences.

\section{Tie-Breaking Rule 7}

If $u_{i}\left(\pi^{0}\right)=u_{i}\left(\pi^{1}\right)$, individual $i$ will vote for the counter-proposal $\pi^{0}$.

\section{Tie-Breaking Rule 8}

If one half of the society votes in favor of $\pi^{0}$ and the other half in favor of $\pi^{1}$, the counter-proposal $\pi^{0}$ will bes implemented.

\section{Tie-Breaking Rule 9}

If $A S_{w}$ is indifferent between a proposal $\pi^{0} \in \Pi$ involving $g\left(\pi^{0}\right)=1$ and a proposal $\pi^{\prime 0} \in \Pi$ involving $g\left(\pi^{\prime 0}\right)=0$, she will always suggest $\pi^{0}$.

\footnotetext{
${ }^{28}$ We assume that $\mathcal{A}$ satisfies some regularity conditions so that $A\left(V_{w}, V_{l}, k, p\right) \neq \emptyset$ if and only if $D\left(V_{w}, V_{l}, k, p\right) \neq \emptyset$.

${ }^{29}$ It is always possible to choose parameter values so that two projects with the desired properties exist.
} 
For this modified two-round institution we prove that, whenever $\hat{p} \leq \frac{1}{2}$, the equilibrium outcome yields that the project is implemented, regardless of $\hat{p}$ being lower than $p$ or not.

\section{Proof of Proposition 4:}

Let $\pi^{1} \in \Pi$ be an arbitrary proposal suggested by $A S_{l}$ and let $\pi^{0} \in \Pi$ be the amendment proposed by $A S_{w}$. Analogously to the case where $A S_{w}$ proposes first, we can define, for each $i \in[0,1]$,

$$
\sigma_{i}\left(g\left(\pi^{0}\right)\right):=s_{i}\left(\pi^{1}\right)+\left(g\left(\pi^{1}\right)-g\left(\pi^{0}\right)\right)\left(v_{i}-k\right)-\left(\int_{0}^{1} s_{j}\left(\pi^{1}\right) d j-\int_{0}^{1} s_{j}\left(\pi^{0}\right) d j\right)
$$

which amounts to the minimum subsidy that has to be given to individual $i$ so that she will vote for $\pi^{0}$ instead of $\pi^{1}$, for given $\pi^{1}$ and $\left(s_{j}\left(\pi^{0}\right)\right)_{j \in[0,1] \backslash\{i\}}$.

Following the argument in the proof of Lemma 2 in Appendix B, we next split the set $(0,1)$ of all individuals except the two agenda-setters into two subsets. For that purpose, we define ${ }^{30}$

$$
\begin{aligned}
& \text { - } \Gamma_{0}:=\arg \min _{Q \subseteq(0,1)}\left\{\int_{Q} \sigma_{j}(0) d j \mid q=\frac{1}{2}\right\}, \\
& \text { - } \Gamma_{1}:=\arg \min _{Q \subseteq(0,1)}\left\{\int_{Q} \sigma_{j}(1) d j \mid q=\frac{1}{2}\right\} .
\end{aligned}
$$

In words, $\Gamma_{0}$ is composed of all sets $Q_{0} \subseteq(0,1)$ of measure $\frac{1}{2}$ containing individuals $j$ with smallest aggregate $\sigma_{j}(0)$, whereas $\Gamma_{1}$ is composed of all sets of $Q_{1} \subseteq(0,1)$ of measure $\frac{1}{2}$ containing individuals $j$ with smallest aggregate $\sigma_{j}(1) .{ }^{31}$ Analogously to the proof of Lemma 2 in Appendix B, $Q_{0} \in \Gamma_{0}$ and $Q_{1} \in \Gamma_{1}$ independently of the exact design of the subsidy scheme $s\left(\pi^{1}\right)$. Also, for some choices of $\pi^{0}$, elements $Q_{0}, Q_{0}^{\prime} \in \Gamma_{0}$ (or $Q_{1}, Q_{1}^{\prime} \in \Gamma_{1}$ ) may differ even in a subset of individuals of positive measure. However, as before, it will transpire that outcomes of the game will be independent of a particular choice of $Q_{0}$ and $Q_{1}$. We select two arbitrary elements $Q_{0} \in \Gamma_{0}$ and $Q_{1} \in \Gamma_{1}$.

This time, we further split set $Q_{0}$ into two disjoint subsets $Q_{0}^{w}$ and $Q_{0}^{l}$, where

$$
\begin{aligned}
& Q_{0}^{w}:=\left\{j \in Q_{0} \mid j \in(0, p]\right\}, \\
& Q_{0}^{l}:=\left\{j \in Q_{0} \mid j \in(p, 1)\right\} .
\end{aligned}
$$

\footnotetext{
${ }^{30}$ Analogous precisions to those made in Footnote 17 need to be made to properly define $\Gamma_{0}$ and $\Gamma_{1}$.

${ }^{31}$ Notice that now, for each $Q_{0} \in \Gamma_{0}$ and $Q_{1} \in \Gamma_{1}$, it holds that $q_{0}=q_{1}=\frac{1}{2}$ and that, as before, $A S_{w}$ and $A S_{l}$ do not belong to $Q_{0} \cup Q_{1}$ by definition.
} 
Set $Q_{0}^{w}$ comprises all winners from $Q_{0}$, and set $Q_{0}^{l}$ all losers from $Q_{0}$. By construction, $Q_{0}=Q_{0}^{w} \cup Q_{0}^{l}$ and $Q_{0}^{w} \cap Q_{0}^{l}=\emptyset$, so $q_{0}^{w}+q_{0}^{l}=q_{0}=\frac{1}{2}$. Moreover, since $p<\frac{1}{2}$, we have $q_{0}^{l}>0$.

Analogously to Lemma 1, it can be proved that, due to Tie-Breaking Rules 7 and 8, an optimal counter-proposal $R\left(\pi^{1}\right)$ will always yield $I\left(\pi^{1}, R\left(\pi^{1}\right)\right)=1$. Moreover, analogously to Lemma 3 in Appendix $\mathrm{B}$, the reaction function of $A S_{w}$, who is now the second agenda-setter, can be summarized, due to Tie-Breaking Rule 9, as follows:

$$
R\left(\pi^{1}\right)= \begin{cases}\tilde{\tilde{\pi}}^{0} & \text { if } u_{0}\left(\tilde{\tilde{\pi}}^{0}\right) \geq u_{0}\left(\tilde{\pi}^{0}\right) \\ \tilde{\pi}^{0} & \text { otherwise }\end{cases}
$$

where

$$
g\left(\tilde{\pi}^{0}\right)=0, \quad s_{j}\left(\tilde{\pi}^{0}\right)= \begin{cases}\max \left\{0, \sigma_{j}(0)\right\} & \text { for } j \in Q_{0}, \\ 0 & \text { for all other } j\end{cases}
$$

and

$$
g\left(\tilde{\tilde{\pi}}^{0}\right)=1, \quad s_{j}\left(\tilde{\tilde{\pi}}^{0}\right)= \begin{cases}\max \left\{0, \sigma_{j}(1)\right\} & \text { for } j \in Q_{1}, \\ 0 & \text { for all other } j .\end{cases}
$$

We point out that, although the subsidy schemes of $\tilde{\pi}^{0}$ and $\tilde{\pi}^{0}$ are only implicitly defined, they are in fact well-defined. ${ }^{32}$

Next, we study the optimal strategy for the first agenda-setter, $A S_{l}$. We claim (and prove below) that, under Tie-Breaking Rule 9, it is always optimal for $A S_{w}$ to choose $\tilde{\pi}^{0}$ instead of $\tilde{\pi}^{0}$ as a counter-proposal to $\pi^{1}$ whenever

$$
\hat{p} \leq \frac{1}{2}
$$

Since $A S_{l}$ can advance $A S_{w}$ 's behavior, we now apply backward induction to analyze the optimal choice for $A S_{l}$. Under $\tilde{\pi}^{0}$, the net utility change for $A S_{l}$ is

$$
u_{1}\left(\tilde{\pi}^{0}\right)=\left(V_{l}-k\right)-\int_{Q_{1}} \max \left\{0, \sigma_{j}(1)\right\} d j \leq V_{l}-k .
$$

As a consequence, $A S_{l}$ 's optimal strategy is to propose $\pi^{1}$ such that $\max \left\{0, \sigma_{j}(1)\right\}=0$ for all $j \in Q_{1}$. This can be achieved by choosing $\pi^{1}$ with $g\left(\pi^{1}\right)=1$ and $s_{j}\left(\pi^{1}\right)=0$ for all $j \in[0,1]$. For this particular choice of $\pi^{1}$, we obtain $Q_{1}=\left[\frac{1}{2}, 1\right), \int_{Q_{1}} \max \left\{0, \sigma_{j}(1)\right\} d j=$ 0 and $u_{1}\left(\tilde{\tilde{\pi}}^{0}\right)=V_{l}-k$. In particular, we conclude that the public project will be

\footnotetext{
B.

${ }^{32}$ See Remark 1 for the analogous definition problem solved in the proof of Lemma 3 in Appendix
} 
implemented if (27) holds, even if doing so is socially inefficient. More specifically, observe that inefficiencies of type 1 and possibly type 3 may arise.

It only remains to prove the above claim. We assume that

$$
u_{i}\left(\tilde{\pi}^{0}\right) \geq u_{i}\left(\pi^{1}\right)
$$

for all $i \in Q_{0}$. We note that if (29) does not hold for a subset of non-zero measure of $Q_{0}$, it must necessarily be the case that $I\left(\pi^{1}, \tilde{\pi}^{0}\right)=0$ due to the way $\tilde{\pi}^{0}$ is constructed. In that case, $\tilde{\pi}^{0}$ cannot be the best response, so $A S_{w}$ 's best response is $\tilde{\tilde{\pi}}^{0}$, as claimed. We distinguish two cases, $q_{0}^{w}>0$ and $q_{0}^{w}=0 .{ }^{33}$

- Case 1: $q_{0}^{w}>0$

Let $\tilde{\tilde{\pi}}^{\prime} 0 \in \Pi$ be a proposal that comprises $g\left(\tilde{\tilde{\pi}}^{\prime}\right)=1$ and the following welldefined $^{34}$ subsidy scheme:

$$
s_{j}\left(\tilde{\tilde{\pi}}^{\prime} 0\right)= \begin{cases}\max \left\{0, \sigma_{j}(0)\right\}+\frac{\frac{1}{2}+q_{0}^{w}-\hat{p}}{q_{0}^{w}}\left(V_{w}-V_{l}\right) & \text { for } j \in Q_{0}^{w}, \\ \max \left\{0, \sigma_{j}(0)\right\}+V_{w}-V_{l} & \text { for } j \in Q_{0}^{l}, \\ 0 & \text { for all other } j .\end{cases}
$$

Notice that if (27) holds the above scheme defines non-negative subsidies for all citizens. Note also that in general $\tilde{\tilde{\pi}}^{\prime 0}$ need not coincide with $\tilde{\tilde{\pi}}^{0}$. The aggregate amount of subsidies under proposal $\tilde{\pi}^{\prime} 0$ is

$$
\begin{aligned}
\int_{0}^{1} s_{j}\left(\tilde{\tilde{\pi}}^{\prime} 0\right) d j & =\int_{j \in Q_{0}} \max \left\{0, \sigma_{j}(0)\right\} d j+\left(V_{w}-V_{l}\right)(1-\hat{p}) \\
& =\int_{j \in Q_{0}} \max \left\{0, \sigma_{j}(0)\right\} d j+V_{w}-k
\end{aligned}
$$

where the second equality holds by the definition of $\hat{p}$.

Next we show that $u_{i}\left(\tilde{\tilde{\pi}}^{\prime}\right) \geq u_{i}\left(\pi^{1}\right)$ for all $i \in Q_{0}$. Since by construction $Q_{0}$ includes half of the society, Tie-Breaking Rules 7 and 8 imply that $\tilde{\pi}^{\prime} 0$ would win

\footnotetext{
${ }^{33}$ In this Proposition we do not need to distinguish between $n \geq \frac{1}{2}$ and $n<\frac{1}{2}$.

${ }^{34}$ An argument similar to that in Remark 1 proves that this subsidy scheme is indeed well-defined.
} 
a vote against $\pi^{1}$. On the one hand, consider $i \in Q_{0}^{w}$. Then,

$$
\begin{aligned}
& u_{i}\left(\tilde{\tilde{\pi}}^{\prime}\right) \\
& =V_{w}-k+\frac{\frac{1}{2}+q_{0}^{w}-\hat{p}}{q_{0}^{w}}\left(V_{w}-V_{l}\right)+\max \left\{0, \sigma_{i}(0)\right\}-\int_{j \in Q_{0}} \max \left\{0, \sigma_{j}(0)\right\} d j-\left(V_{w}-k\right) \\
& =\frac{\frac{1}{2}+q_{0}^{w}-\hat{p}}{q_{0}^{w}}\left(V_{w}-V_{l}\right)+\max \left\{0, \sigma_{i}(0)\right\}-\int_{j \in Q_{0}} \max \left\{0, \sigma_{j}(0)\right\} d j \\
& >\max \left\{0, \sigma_{i}(0)\right\}-\int_{j \in Q_{0}} \max \left\{0, \sigma_{j}(0)\right\} d j=u_{i}\left(\tilde{\pi}^{0}\right) \geq u_{i}\left(\pi^{1}\right),
\end{aligned}
$$

where the last inequality holds by (29) since $i \in Q_{0}$. On the other hand, consider $i \in Q_{0}^{l}$. Then,

$$
\begin{aligned}
u_{i}\left(\tilde{\tilde{\pi}}^{\prime}\right) & =\left(V_{l}-k\right)+\left(V_{w}-V_{l}\right)+\max \left\{0, \sigma_{i}(0)\right\}-\int_{j \in Q_{0}} \max \left\{0, \sigma_{j}(0)\right\} d j-\left(V_{w}-k\right) \\
& =\max \left\{0, \sigma_{i}(0)\right\}-\int_{j \in Q_{0}} \max \left\{0, \sigma_{j}(0)\right\} d j=u_{i}\left(\tilde{\pi}^{0}\right) \geq u_{i}\left(\pi^{1}\right),
\end{aligned}
$$

where the last inequality holds by (29) since $i \in Q_{0}$.

Lastly, notice that $A S_{w}$ is indifferent between $\tilde{\pi}^{\prime} 0$ and $\tilde{\pi}^{0}$. Indeed,

$$
u_{0}\left(\tilde{\pi}^{\prime}\right)=-\int_{j \in Q_{0}} \max \left\{0, \sigma_{j}(0)\right\} d j=u_{0}\left(\tilde{\pi}^{0}\right) .
$$

- Case 2: $q_{0}^{w}=0$

In this second case, let $\tilde{\tilde{\pi}}^{\prime 0} \in \Pi$ be a proposal that comprises $g\left(\tilde{\tilde{\pi}}^{\prime}\right)=1$ and the following subsidy scheme:

$$
s_{j}\left(\tilde{\tilde{\pi}}^{\prime} 0\right)= \begin{cases}\left(V_{w}-V_{l}\right)+\max \left\{0, \sigma_{j}(0)\right\} & \text { for } j \in Q_{0}^{l}, \\ 0 & \text { for all other } j .\end{cases}
$$

Notice again that in general $\tilde{\tilde{\pi}}^{\prime 0}$ need not coincide with $\tilde{\tilde{\pi}}^{0}$. The aggregate amount of subsidies under proposal $\tilde{\tilde{\pi}}^{\prime 0}$ is now

$$
\int_{0}^{1} s_{j}\left(\tilde{\tilde{\pi}}^{\prime}\right) d j=\int_{j \in Q_{0}} \max \left\{0, \sigma_{j}(0)\right\} d j+\frac{1}{2}\left(V_{w}-V_{l}\right) .
$$

As in Case 1 , we show that $u_{i}\left(\tilde{\tilde{\pi}}^{\prime}\right) \geq u_{i}\left(\pi^{1}\right)$ for all $i \in Q_{0}$, so, given that $Q_{0}$ includes half of the society, Tie-Breaking Rules 7 and 8 imply that $\tilde{\tilde{\pi}}^{\prime}{ }^{0}$ would be 
chosen over $\pi^{1}$. Let $i \in Q_{0}$. Then,

$$
\begin{aligned}
& u_{i}\left(\tilde{\tilde{\pi}}^{\prime}\right) \\
& =\left(V_{l}-k\right)+\left(V_{w}-V_{l}\right)+\max \left\{0, \sigma_{i}(0)\right\}-\int_{j \in Q_{0}} \max \left\{0, \sigma_{j}(0)\right\} d j-\frac{1}{2}\left(V_{w}-V_{l}\right) \\
& =\left(\frac{1}{2}-\hat{p}\right)\left(V_{w}-V_{l}\right)+\max \left\{0, \sigma_{i}(0)\right\}-\int_{j \in Q_{0}} \max \left\{0, \sigma_{j}(0)\right\} d j \\
& \geq \max \left\{0, \sigma_{i}(0)\right\}-\int_{j \in Q_{0}} \max \left\{0, \sigma_{j}(0)\right\} d j=u_{i}\left(\tilde{\pi}^{0}\right) \geq u_{i}\left(\pi^{1}\right),
\end{aligned}
$$

where the second equality holds by definition of $\hat{p}$, the first inequality holds since $\frac{1}{2}-\hat{p} \geq 0$, and the last inequality holds since $i \in Q_{0}$.

Lastly, notice that $A S_{w}$ weakly prefers the implementation of $\tilde{\pi}^{\prime}{ }^{0}$ to $\tilde{\pi}^{0}$. Indeed,

$$
\begin{aligned}
u_{0}\left(\tilde{\tilde{\pi}}^{\prime}\right) & =\left(V_{w}-k\right)-\frac{1}{2}\left(V_{w}-V_{l}\right)-\int_{j \in Q_{0}} \max \left\{0, \sigma_{j}(0)\right\} d j \\
& \geq-\int_{j \in Q_{0}} \max \left\{0, \sigma_{j}(0)\right\} d j=u_{0}\left(\tilde{\pi}^{0}\right),
\end{aligned}
$$

where the inequality holds by $(27)$.

Notice that $\tilde{\tilde{\pi}}^{0}$ will win against $\tilde{\pi}^{1}$ and that $u_{0}\left(\tilde{\tilde{\pi}}^{0}\right) \geq u_{0}\left(\tilde{\tilde{\pi}}^{\prime}\right) \geq u_{0}\left(\tilde{\pi}^{0}\right)$ in both Case 1 and Case 2. This holds since $\tilde{\pi}^{0}$ is the optimal response for $A S_{w}$ provided that the project is not implemented and $A S_{w}$ weakly prefers to implement $\tilde{\pi}^{\prime}{ }^{0}$ to $\tilde{\pi}^{0}$. If that were not the case, due to Tie-Breaking Rule 9 we would find that the optimal proposal for $A S_{w}$ would be $\tilde{\tilde{\pi}}^{\prime}$, contradicting the fact that either $\tilde{\pi}^{0}$ or $\tilde{\tilde{\pi}}^{0}$ is her optimal response. Therefore it is optimal for $A S_{w}$ to choose $\tilde{\tilde{\pi}}^{0}$, as we claimed.

\section{C.3 When the tax system is flexible}

In the last part of the paper, the tax schedule is no longer limited to being uniform across voters but is only required to be non-negative. That is, in this section $t(\pi)$ is a function on $[0,1]$ such that $t_{j}(\pi) \geq 0$ for all $j \in[0,1]$ and

$$
\int_{0}^{1} t_{j}(\pi) d j=g(\pi) k+\int_{0}^{1} s_{j}(\pi) d j .
$$




\section{Proof of Proposition 5:}

For a given proposal $\pi \in \Pi$ and a given individual $j \in[0,1]$, we have the following net utility changes:

$$
u_{j}(\pi)=g(\pi) V_{j}+s_{j}(\pi)-t_{j}(\pi)
$$

We assume that

$$
V_{l}>0
$$

and we show that, when (32) holds, inefficiencies may arise. Indeed, consider the proposal $\pi^{*}$ with $g\left(\pi^{*}\right)=1$ and the subsidy and tax schemes given respectively by

$$
s_{j}\left(\pi^{*}\right)= \begin{cases}\hat{s} & \text { for } j \in\left(0, \frac{1}{2}\right], \\ 0 & \text { for all other } j,\end{cases}
$$

and

$$
t_{j}\left(\pi^{*}\right)= \begin{cases}2 k+\hat{s} & \text { for } j \in\left(\frac{1}{2}, 1\right), \\ 0 & \text { for all other } j .\end{cases}
$$

With the above proposal, we obtain

$$
u_{j}\left(\pi^{*}\right)= \begin{cases}V_{w} & \text { for } j=0, \\ V_{w}+\hat{s} & \text { for } j \in(0, p], \\ V_{l}+\hat{s} & \text { for } j \in\left(p, \frac{1}{2}\right] \\ V_{l}-2 k-\hat{s} & \text { for } j \in\left(\frac{1}{2}, 1\right) \\ V_{l} & \text { for } j=1 .\end{cases}
$$

Let $\pi \in \Pi$ be an arbitrary proposal. Then

$$
u_{j}(\pi)=g(\pi) V_{l}+s_{j}(\pi)-t_{j}(\pi) \leq V_{l}+\hat{s}=u_{j}\left(\pi^{*}\right) \text { for all } j \in\left(0, \frac{1}{2}\right] .
$$

Moreover,

$$
u_{0}(\pi)=g(\pi) V_{w}-t_{0}(\pi) \leq V_{w}=u_{0}\left(\pi^{*}\right) .
$$

and

$$
u_{1}(\pi)=g(\pi) V_{l}-t_{1}(\pi) \leq V_{l}=u_{1}\left(\pi^{*}\right)
$$

That is, under $\pi^{*}$ both agenda-setters and all voters $j \in\left(0, \frac{1}{2}\right]$ obtain their maximum possible utility. As a consequence of Tie-Breaking Rules 1, 2, and 3, it immediately follows that there is an equilibrium in which both agenda-setters will propose $\pi^{*}$, leading to public project implementation regardless of whether it is socially efficient, 
coupled with strictly aggregate subsidies. That is, when (32), holds inefficiencies of types 1 and 3 will arise. 


\section{References}

Austen-Smith D. (1987), Sophisticated sincerity: voting over endogenous agendas, American Political Science Review 81(4), 1323-1330.

Aghion P., Bolton P. (2003), Incomplete Social Contracts, Journal of the European Economic Association 1(1), 38-67.

Baron D., Ferejohn J. (1989), Bargaining in Legislatures, The American Political Science Review 83(4), 1181-1206.

Banks J. (1985), Sophisticated voting outcomes and agenda control, Social Choice and Welfare 1, 295-306.

Banks J., Duggan J. (2000), A bargaining model of collective choice, American Political Science Review 94, 73-88.

Banks J., Gasmi F. (1987), Endogenous Agenda Formation in Three Person Committees, Social Choice and Welfare 4, 133-152

Bernheim D., Rangel A., Rayo L. (2006), The Power of the Last Word in Legislative Policy Making, Econometrica 74(5), 1161-1190.

Dirmeier D., Merlo A. (2000), Government Turnover in Parliamentary Democracies, Journal of Economic Theory 94, 46-79.

Dutta B., Jackson M., Le Breton M. (2001), Strategic Candidacy and Voting Procedures, Econometrica 69(4), 1013-1037.

Ferejohn J., Fiorina M., McKelvey R. D. (1987), Voting and Agenda Independence in the Distributive Politics Setting, American Journal of Political Science 31(1), 169-193.

Gersbach H. (2009), Democratic Mechanisms, Journal of the European Economic Association 7(6), 1436-1469.

Gersbach H., Hahn V., and Imhof S. (2012), Tax Rules, Social Choice and Welfare, DOI: $10.1007 / \mathrm{s} 00355-012-0675-1$. 
Jackson M., and Moselle B. (2002), Coalition and Party Formation in a Legislative Voting Game, Journal of Economic Theory 103, 49-87.

Miller N. (1980), A new solution set for tournaments and majority voting: further graph-theoretic approaches to the theory of voting, American Journal of Political Science 24, 68-96

Myerson R. (1993), Incentives to Cultivate Favored Minorities Under Alternative Electoral Systems, The American Political Science Review 87(4), 856-869.

McKelvey R. D. (1976), Intransitivities in multidimensional voting models and some implications for agenda control, Journal of Economic Theory 12, 472-482.

McKelvey R. D. (1979), General conditions for global intransitivities in formal voting models, Econometrica 47, 1085-1111.

Romer T., Rosenthal H. (1978), Political resource allocation, controlled agendas, and the status quo, Public Choice 33, 27-45.

Shepsle K., Weingast B. (1984), Do Rules of Procedure Matter?, The Journal of Politics, 46(1), 206-221. 